Draft VERsion November 6, 2018

Preprint typeset using LATEX style AASTeX6 v. 1.0

\title{
REVISITING THE LICK OBSERVATORY SUPERNOVA SEARCH VOLUME-LIMITED SAMPLE: UPDATED CLASSIFICATIONS AND REVISED STRIPPED-ENVELOPE SUPERNOVA FRACTIONS
}

\author{
IsaAc Shivvers, ${ }^{1}$ Maryam Modjaz, ${ }^{2}$ WeiKang Zheng, ${ }^{1}$ Yuqian Liu, ${ }^{2}$ \\ Alexei V. Filippenko, ${ }^{1}$ Jeffrey M. Silverman,${ }^{3}$ Thomas Matheson, ${ }^{4}$ Andrea Pastorello,${ }^{5}$ \\ Or Graur, ${ }^{6,2,7,8}$ Ryan J. Foley, ${ }^{9}$ Ryan Chornock, ${ }^{10}$ Nathan Smith, ${ }^{11}$ \\ Jesse Leaman, ${ }^{12}$ Stefano Benetti ${ }^{5}$
}

${ }^{1}$ Department of Astronomy, University of California, Berkeley, CA 94720-3411, USA

${ }^{2}$ Center for Cosmology and Particle Physics, New York University, New York, NY 10003, USA

${ }^{3}$ Department of Astronomy, University of Texas at Austin, Austin, TX 78712, USA

${ }^{4}$ National Optical Astronomy Observatory, Tucson, AZ 85719, USA

${ }^{5}$ INAF-Osservatorio Astronomico di Padova, Vicolo dell'Osservatorio 5, I-35122 Padova, Italy

${ }^{6}$ Harvard-Smithsonian Center for Astrophysics, 60 Garden St., Cambridge, MA 02138, USA

${ }^{7}$ Department of Astrophysics, American Museum of Natural History, New York, NY 10024-5192, USA

${ }^{8}$ NSF Astronomy and Astrophysics Postdoctoral Fellow

${ }^{9}$ Department of Astronomy and Astrophysics, University of California, Santa Cruz, CA 95064, USA

${ }^{10}$ Department of Physics and Astronomy, Ohio University, Athens, OH 45701, USA

${ }^{11}$ Steward Observatory, University of Arizona, 933 N. Cherry Ave., Tucson, AZ 85721, USA

${ }^{12}$ Advanced Robotics and Automation Lab, Department of Computer Science and Engineering, University of Nevada, Reno, NV 89557, USA

\section{ABSTRACT}

We re-examine the classifications of supernovae ( $\mathrm{SNe}$ ) presented in the Lick Observatory Supernova Search (LOSS) volume-limited sample with a focus on the stripped-envelope SNe. The LOSS volumelimited sample, presented by Leaman et al. (2011) and Li et al. (2011b), was calibrated to provide meaningful measurements of SN rates in the local universe; the results presented therein continue to be used for comparisons to theoretical and modeling efforts. Many of the objects from the LOSS sample were originally classified based upon only a small subset of the data now available, however, and recent studies have both updated some subtype distinctions and improved our ability to perform robust classifications, especially for stripped-envelope SNe. We re-examine the spectroscopic classifications of all events in the LOSS volume-limited sample (180 SNe and SN impostors) and update them if necessary. We discuss the populations of rare objects in our sample including broad-lined Type Ic SNe, Ca-rich SNe, SN 1987A-like events (we identify SN 2005io as SN 1987A-like here for the first time), and peculiar subtypes. The relative fractions of Type Ia SNe, Type II SNe, and stripped-envelope $\mathrm{SNe}$ in the local universe are not affected, but those of some subtypes are. Most significantly, after discussing the often unclear boundary between SNe Ib and Ic when only noisy spectra are available, we find a higher SN Ib fraction and a lower SN Ic fraction than calculated by Li et al. (2011b): spectroscopically normal SNe Ib occur in the local universe $1.7 \pm 0.9$ times more often than do normal SNe Ic.

Keywords: supernovae; spectroscopy

\section{INTRODUCTION}

The Lick Observatory Supernova Search (LOSS) has been a long-running project at the University of California, Berkeley, using the Katzman Automatic Imaging Telescope at Lick Observatory (KAIT; e.g., Li et al. 2000; Filippenko et al. 2001b; Filippenko 2003,

ishivvers@berkeley.edu
2005), with many spectroscopic follow-up observations obtained with the $3 \mathrm{~m}$ Shane telescope at Lick and the $10 \mathrm{~m}$ telescopes at Keck Observatory. LOSS/KAIT has been discovering and observing $\mathrm{SNe}$ since first light in 1996; these data have contributed to several $\mathrm{PhD}$ theses and formed the foundation of many research projects on SNe. A detailed examination of the relative rates of nearby SNe was one of those projects, and was published as a series of papers in 2011 (Leaman et al. 2011; Li et al. 
2011a,b; Maoz et al. 2011; Smith et al. 2011a). The second of these, Li et al. (2011b, L11 hereafter), presents a sample of 180 events that occurred within $80 \mathrm{Mpc}$ (for Type Ia SNe) or $60 \mathrm{Mpc}$ (for core-collapse SNe), all of which were spectroscopically classified (the classes of SNe are differentiated primarily via spectroscopy; e.g., Filippenko 1997). Most SN classifications from this time period were performed via visual inspection and comparisons with spectra of a few SNe of well-understood types and subtypes.

Over time we have found that a small fraction of the objects in L11 deserve reclassification; in some cases this is because the original classifications were made using only a subset of the now-available data on the objects, while in other cases our more modern classification methods are less prone to errors than the methods used at the time of classification. Independent of data quality or cadence, there is a history of debate in the literature over the exact distinction (if any) between SNe Ib and SNe Ic and whether transitional events showing weak helium lines exist (e.g., Filippenko et al. 1990a; Wheeler \& Harkness 1990; Wheeler et al. 1994; Clocchiatti et al. 1996; Matheson et al. 2001; Branch et al. 2006).

The results of recent efforts by Liu \& Modjaz (2014), Modjaz et al. (2014), and Liu et al. (2016) argue that the distinction between SNe Ib and SNe Ic is useful, and they offer a clearly defined scheme for discriminating between them alongside updated software tools to perform those classifications in a repeatable manner. Modjaz et al. (2014) identify as SNe Ib all events with detections of both the He I $\lambda 6678$ and He I $\lambda 7065$ lines at phases between maximum light and $\sim 50$ days post-maximum, regardless of line strengths (the stronger He I $\lambda 5876$ line is also present, but overlaps with $\mathrm{Na}$ I). They find that at least one good spectrum observed at these phases is necessary and sufficient to detect the helium lines, which are often absent at pre-maximum and nebular phases even for helium-rich events. Using this classification scheme, they find evidence for a transitional population of "weak helium" SNe Ib (Valenti et al. 2011; Modjaz et al. 2014; Liu et al. 2016).

Clarifying the distinction between $\mathrm{SNe} \mathrm{Ib}$ and SNe Ic is important given the surprising ratio of population fractions for these subtypes found by LOSS $\left(\mathrm{SNe} I c / \mathrm{SNe} \mathrm{Ib}=14.9_{-3.8}^{+4.2} \% / 7.1_{-2.6}^{+3.1} \%\right.$; Smith et al. 2011a), which has proven difficult to reproduce with stellar modeling efforts (e.g., Georgy et al. 2009; Yoon et al. 2010; Yoon 2015), though see also Groh et al. (2013a). Modjaz et al. (2014) show that a subset of the objects originally labeled SNe Ic in their sample in fact do qualify for the SN Ib label according to the definition above, and so they relabel these events as SNe Ib (see their discussion of all such cases, in their $\S 4.2$ ).

For some of those SNe, the spectra that were used to classify them and thus announce their types were obtained before the helium lines became prominent; for some, applying proper telluric corrections made the He I $\lambda 6678$ or $\lambda 7065$ lines more apparent; for others the spectra show clear helium but the exact division between $\mathrm{SNe} \mathrm{Ib}$ and SNe Ic was under debate in the literature at the time of classification (e.g., SN 1990U; Filippenko et al. 1990b; Filippenko \& Shields 1990; Matheson et al. 2001). We explore these issues within the LOSS sample and also find that some events with helium lines were systematically labeled as SNe Ic — we update the classifications for these events and recalculate the relative fractions of core-collapse events.

In this article we re-examine the classifications of the 180 events in the volume-limited sample of L11 and we make public all spectra of them we have been able to locate. This work was performed in conjunction with Graur et al. (2016a,b), who re-examine correlations between SN rates and galaxy properties. Note that much of the spectroscopy discussed herein has already been described in the literature and made publicly available by, for example, Silverman et al. (2012, SNe Ia), Faran et al. (2014a,b, SNe II), Matheson et al. (2001, SNe IIb/Ib/Ic). We collect these spectra, light curves obtained by LOSS, as-yet unpublished spectra from our archives, and as-yet unpublished spectra contributed from other SN research groups' archives, and analyze the complete set.

We present 151 newly published spectra of $71 \mathrm{SNe}$ and 20 rereduced KAIT light curves. In $\S 2$ we describe these data, in $\S 3$ we detail our methods for classification, in $\S 4$ we present all updated classifications and discuss notable events within the sample, in $\S 5$ we calculate updated core-collapse $\mathrm{SN}$ rates in the local universe, in $\S 6$ we discuss the implications these updates have for our understanding of the progenitors of stripped-envelope SNe, and in $\S 7$ we conclude.

\section{DATA}

Spectra were collected from our own UC Berkeley Supernova Database (UCB SNDB; Silverman et al. 2012), ${ }^{1}$ from the literature, and from WISeREP (the Weizmann Interactive Supernova Data REPository; Yaron \& GalYam 2012). ${ }^{2}$ We do not include any results from spectropolarimetric or nonoptical observations; we know of no such observations that would help for the few events we cannot robustly classify using optical data. We made an effort to track down as-yet unpublished spectra for all objects with sparse or no spectral data in our database

\footnotetext{
1 The SNDB was updated in 2015 and is available online at http://heracles astro berkeley . edu/sndb/.

2 http://wiserep.weizmann.ac.il/
} 
or in the public domain. All objects in this sample were classified in the Central Bureau of Electronic Telegrams (CBETs), and we contacted original authors to request data whenever possible. Contributions were made by the Center for Astrophysics (CfA) SN group (Matheson et al. 2008), ${ }^{3}$ the Padova-Asiago SN group (Tomasella et al. 2014), ${ }^{4}$ the Carnegie Supernova Project (CSP; Hamuy et al. 2006), ${ }^{5}$ and the National Astronomical Observatories, Chinese Academy of Sciences (NAOC) SN group (Qui et al. 1999; Li et al. 1999).

We publish spectra from the following observatories and instruments:

- the Kast double spectrograph (Miller \& Stone 1993) mounted on the Shane $3 \mathrm{~m}$ telescope at Lick Observatory;

- the Low Resolution Imaging Spectrometer (LRIS; Oke et al. 1995) and the Echellette Spectrograph and Imager (ESI; Sheinis et al. 2002) on the $10 \mathrm{~m}$ Keck I \& II telescopes at Keck Observatory;

- the FAST spectrograph (Fabricant et al. 1998) on the Tillinghast 60 inch telescope and the Blue Channel spectrograph (Schmidt et al. 1989) on the $6.5 \mathrm{~m}$ Multiple Mirror Telescope (MMT) at the Fred Lawrence Whipple Observatory (FLWO);

- the European Southern Observatory (ESO) Faint Object Spectrograph and Camera (EFOSC; Buzzoni et al. 1984) on the ESO $3.6 \mathrm{~m}$ telescope, the Danish Faint Object Spectrograph and Camera (DFOSC, modeled after EFOSC; Andersen et al. 1995) on the Danish $1.54 \mathrm{~m}$ telescope, and the ESO Multi-Mode Instrument in medium resolution spectroscopy mode (EMMI; Dekker et al. 1986) on the ESO $3.58 \mathrm{~m}$ New Technology Telescope, all at La Silla Observatory;

- the Asiago Faint Object Spectrograph and Camera (AFOSC, modeled after EFOSC) on the $1.82 \mathrm{~m}$ Copernico telescope and the Boller and Chivens spectrograph $\left({\left.\mathrm{B} \& \mathrm{C}_{1.2}\right)}\right)$ on the $1.2 \mathrm{~m}$ Galileo telescope at Asiago Observatory;

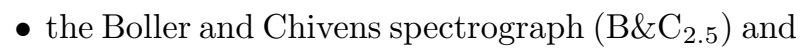
the Wide Field Reimaging CCD Camera in longslit spectroscopy mode (WFCCD, described by Hamuy et al. 2006) on the $2.5 \mathrm{~m}$ du Pont telescope and the Low Dispersion Survey Spectrograph (LDSS-2; Allington-Smith et al. 1994) on

\footnotetext{
3 https://www.cfa.harvard.edu/supernova/

4 http://sngroup.oapd.inaf.it/

5 http://csp.obs.carnegiescience.edu/
}

the $6.5 \mathrm{~m}$ Magellan Clay telescope at Las Campanas Observatory;

- and the Optomechanics Research, Inc. ${ }^{6}$ spectrograph (OMR) mounted on the NAOC $2.16 \mathrm{~m}$ telescope at Xinglong Observatory near Beijing, China.

Details of the spectral reduction pipeline used by the UCB team are described by Silverman et al. (2012). Matheson et al. (2008), Blondin et al. (2012), and Modjaz et al. (2014) discuss the reduction process performed on the CfA spectra, and Hamuy et al. (2006) outline the reduction process performed on the CSP spectra. Standard IRAF ${ }^{7}$ reduction packages were used by the Padova-Asiago and NAOC groups. Most spectra presented here have resolutions of $\sim 10 \AA$, were observed at or near the parallactic angle (Filippenko 1982), and were flux calibrated with bright standard stars observed at similar airmasses. Most spectra have also been corrected for wavelength-dependent telluric absorption. Details of the observations and data-reduction methods vary from group to group, and we discuss any possible data-quality issues for the spectra most vital to our classification effort in the text.

All photometry used by L11 and in this effort was observed at Lick Observatory with KAIT or the Nickel $1 \mathrm{~m}$ telescope, and all SNe discussed here were discovered by LOSS/KAIT (e.g., Li et al. 2000; Filippenko et al. 2001b). KAIT photometry is generally performed on unfiltered images (the clear band), though filtered BVRI KAIT images of some events are available. Nickel data are observed through a BVRI filter set. Details for both instruments and for our photometry reduction pipeline are given by Ganeshalingam et al. (2010), and we present these light curves as observed, without correcting for Milky Way (MW) or host-galaxy dust absorption, unless otherwise stated. All of the spectra and photometry used in this project will be made public through the UCB SNDB, WiseREP, and the Open Supernova Cata$\log$ (Guillochon et al. 2016). ${ }^{8}$ See Appendix A for logs of the data released publicly here for the first time.

\section{CLASSIFICATION METHODS}

Following Silverman et al. (2012) and Modjaz et al. (2014), we use the SN IDentification code $^{9}$ (SNID; Blondin \& Tonry 2007) as our primary classification

\footnotetext{
6 http://www. echellespectrographs. com/about.htm

7 http://iraf.noao.edu/

8 https://sne.space/

9 http://people.lam.fr/blondin.stephane/software/snid/ index.html
} 
tool. SNID classifies SNe by cross-correlating an input (optical) spectrum against a library of template spectra (Tonry \& Davis 1979). Updated sets of template spectra have been released since the original release of SNID for this study we use the BSNIP v7.0 templates (Silverman et al. 2012) augmented by the Liu \& Modjaz (2014) stripped-envelope templates (and following all suggestions from their Table 4). When running SNID, we set the SN redshift with the forcez keyword using observed host-galaxy redshifts from the NASA/IPAC Extragalactic Database (NED). ${ }^{10}$ For those SNe that SNID alone cannot identify, we incorporate results from two other spectral identification codes, Superfit ${ }^{11}$ (Howell et al. 2005) and GELATO ${ }^{12}$ (Harutyunyan et al. 2008), and for some stripped-envelope $\mathrm{SNe}$ we also compare to the average spectra of Liu et al. (2016).

As shown by (for example) L11, the light curves of $\mathrm{SNe} \mathrm{Ic}$, Ib, and IIb are similar to each other, but are generally distinguishable from those of SNe Ia and hydrogen-rich core-collapse SNe. We incorporate lightcurve information in our classifications when it proves useful, comparing the light curves of individual objects to the clear-band templates from L11 and providing constraints on the phases of spectra. Recent studies have advanced our understanding of stripped-envelope SN light-curve evolution (e.g., Drout et al. 2011; Cano 2013; Bianco et al. 2014; Taddia et al. 2015; Lyman et al. 2016; Prentice et al. 2016). Drout et al. (2011) present template $\mathrm{SN} \mathrm{Ib/c} \mathrm{light} \mathrm{curves} \mathrm{in} \mathrm{the} R$ and $V$ bands assembled from 25 events and Lyman et al. (2016) give template bolometric light curves assembled from 38 events, while L11 produce four template light curves for stripped-envelope SNe: "Ibc.fast," "Ibc.ave," "Ibc.slow," and "IIb." The Ibc.ave template is very similar to the $R$-band SN Ibc template of Drout et al. (2011) and the SN Ib and SN Ic templates of Lyman et al. (2016). The SN IIb templates from L11 and Lyman et al. (2016) are also in good agreement, and both show cooling envelope emission followed by a dip and a rise to a second radioactively-powered peak, with a postpeak evolution basically indistinguishable from that of $\mathrm{SNe} \mathrm{Ib} / \mathrm{c}$. L11 do not produce a template for broad-lined Type Ic SNe (labeled SNe Ic-BL here), but other authors show that SN Ic-BL light curves are quite similar to those of other $\mathrm{SNe} \mathrm{Ib} / \mathrm{c}$ though trending toward higher absolute luminosities (e.g., Drout et al. 2011; Taddia et al. 2015; Prentice et al. 2016).

Several recent large-scale SN data releases have re-

\footnotetext{
10 https://ned.ipac.caltech.edu/

11 http://www.dahowell. com/superfit.html

12 https://gelato.tng.iac.es/
}

lied on SNID classifications using relatively stringent requirements for a robust identification, requiring a high rlap value for the top match (rlap is a quality parameter used by SNID - a higher value corresponds to a more trustworthy classification) and that the first few matches be of the same subtype (e.g., Silverman et al. 2012; Graur \& Maoz 2013; Modjaz et al. 2014; Graur et al. 2015). We follow these methods whenever possible, and for most of the SNe in our sample they clearly indicate a single type and subtype.

All of the SNe Ia in this sample have been examined in detail by other authors (e.g., Blondin et al. 2012; Silverman et al. 2012; Folatelli et al. 2013). We follow the methods of Silverman et al. (2012) to determine SN Ia subtypes, and we do not attempt to identify subpopulations within the normal SNe Ia - i.e., high-velocity events (Wang et al. 2009a) or the subgroups defined by Benetti et al. (2005). We discuss the more peculiar SNe Ia from this sample in $\S 4.3$.

We do not attempt to sort the hydrogen-rich SNe II into the IIP and IIL subtypes. Type II SNe have long been sorted into those that exhibit a clear plateau phase and those that decline linearly in magnitudes (IIP and IIL, respectively; e.g., Barbon et al. 1979; Filippenko 1997). L11 used spectra to identify H-rich SNe, and then labeled as Type IIL those that decline more than $0.5 \mathrm{mag}$ in the $R$ band during the first $50 \mathrm{~d}$ after explosion and the rest as IIP, but recent work has shown that the issue may be more complex. While Arcavi et al. (2012) find there to be distinct SN IIP and SN IIL subclasses among the $R$-band light curves of $21 \mathrm{H}$-rich noninteracting Type II SNe, Anderson et al. (2014) show that their sample of $V$-band light curves for $116 \mathrm{SNe}$ II indicate that there is a continuous distribution of properties for these events. Rubin et al. (2016) present an analysis of the early light curve rise for 57 events finding only a weak correlation between rise times and decline rates, Sanders et al. (2015) and Valenti et al. (2016) argue that there exists a continuous distribution of properties for SNe II and that there is no evidence for separate SNe IIP and SNe IIL subclasses, while Rubin \& GalYam (2016) argue for a type II subclassification system based upon both the rise and the fall of the light curves and Faran et al. (2014a) argue that a simple subclass definition based upon the light curve decline alone remains reasonable. Throughout this article, we group the SN IIP-like and SN IIL-like events under the label "SNe Type II," but when comparing to data from other sources we preserve the SN IIP/SN IIL labels if given by the original authors.

Though we do not differentiate between SNe IIL and SNe IIP, we do identify other H-rich subclasses. We identify the H-rich SNe with narrow spectral emission lines indicative of interaction with dense circumstellar 
material (SNe IIn; e.g., Schlegel 1990; Chugai 1991; Filippenko 1991) and the SN impostors, thought to be nonterminal ejections or explosions from the surface of massive stars (labeled "IIni" in L11; e.g., Van Dyk et al. 2000; Maund et al. 2006; Smith et al. 2011b). We also identify three slow-rising (SN 1987A-like) Type II SNe in our sample (e.g., Arnett et al. 1989; McCray 1993).

Our main focus is on the stripped-envelope SNe. We divide this class into those with some hydrogen features (SNe IIb), those without hydrogen features (or with only very weak hydrogen features; Liu et al. 2016) but with clear helium features (SNe Ib), and those exhibiting neither clear hydrogen nor clear helium features (SNe Ic). The exact distinction between $\mathrm{SNe} \mathrm{Ib}$ and Ic has been an issue of some debate in the literature; we follow Modjaz et al. (2014) and Liu et al. (2016) to define the differences between these subclasses. We differentiate between Type Ic SNe and Ic-BL SNe (see Modjaz et al. 2016), and we identify the "calcium-rich" SNe separately (included in the Ibc-pec category by L11, this class of events has been described by, e.g., Filippenko et al. 2003; Perets et al. 2010; Kasliwal et al. 2012).

For events with spectra that match both the $\mathrm{SN} \mathrm{Ib}$ and SN Ic SNID templates equally well, we discuss the available data in detail and assign the label "Ib/Ic" (i.e., unsure) if we remain unable to determine a single best classification. Figure 1 shows that this classification scheme tends to move events that were previously labeled $\mathrm{SNe}$ Ic into the $\mathrm{Ib}$ or $\mathrm{Ib} / \mathrm{Ic}$ categories (there are no $\mathrm{SNe}$ Ib that we reclassify as SNe Ic in this work).

We follow Smith et al. (2011a) and group the SNe IIb with other stripped-envelope $\mathrm{SNe}$ in our sample, although L11 included them with the Type II SNe. SNe IIb show a strong $\mathrm{H} \alpha$ line at early times, as do normal SNe II, but then the hydrogen fades and the later spectra of SNe IIb resemble those of SNe Ib (e.g., Filippenko 1988; Filippenko et al. 1993; Filippenko 1997; Pastorello et al. 2008; Chornock et al. 2011; Milisavljevic et al. 2013). Several authors have claimed the detection of weak high-velocity hydrogen features in SNe Ib and Ic (e.g., Branch et al. 2006; Parrent et al. 2007, 2016; Liu et al. 2016). However, Liu et al. (2016) argue that the putative weak $\mathrm{H} \alpha$ absorption line often present in $\mathrm{SNe} \mathrm{Ib}$ is, at all phases, weaker than the $\mathrm{H} \alpha$ line in SNe IIb, and that SNID capably distinguishes between $\mathrm{SNe} \mathrm{Ib}$ and $\mathrm{SNe}$ IIb even after the strong $\mathrm{H} \alpha$ feature of the SNe IIb has faded, so long as spectra were obtained during the photospheric phase. The nebular spectra of SNe IIb and $\mathrm{SNe} \mathrm{Ib}$, on the other hand, are often very similar and are not well separated by SNID. When discriminating between Types IIb and Ib, we trust the SNID result if obtained from spectra of the photospheric phase.

To examine possible biases SNID may introduce when classifying stripped-envelope subtypes, we ran a series of

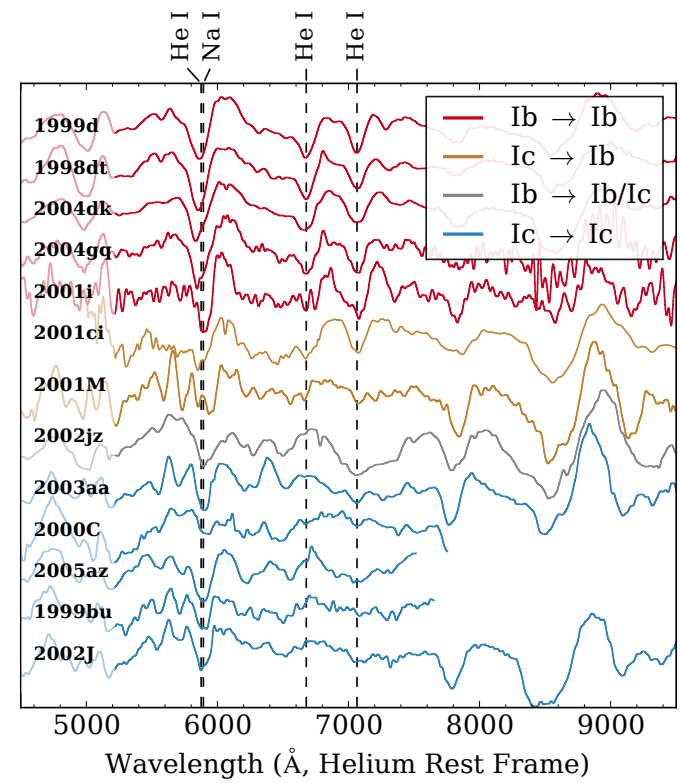

Figure 1. Representative sample of spectra of the $\mathrm{SNe} \mathrm{Ib}$ and Ic in our sample, observed between 5 and 20 days after peak brightness, as well as spectra of three SNe for which we provide updated classifications (SNe 2001M, 2001ci, and 2002jz). We have subtracted a spline continuum from these spectra, smoothed them with a $50 \AA$ Gaussian kernel, and shifted them in velocity space to align their He I features (or $\mathrm{Na}$ I $\lambda 5892$ if no helium is detected).

trials introducing wavelength restrictions, noise, and artificial dust reddening to spectra of Type IIb, Ib, and Ic $\mathrm{SNe}$ at two different phases in their evolution (near maximum brightness and 2-4 weeks post-maximum). We classified the degraded spectra with the methods described above and compared the results to those obtained from the original data. We chose events that are not included in the SNID template set and for which we have relatively high signal-to-noise ratio $(\mathrm{S} / \mathrm{N})$ spectra $(\mathrm{S} / \mathrm{N}>30)$ at these phases covering $\sim 3500-10,000 \AA$ : SN IIb 2003ed, SN Ib 1998dt, and SN Ic 2003aa. The spectra used in this study either cover a similar wavelength range or $\sim 3500-7500 \AA$; we test the efficacy of SNID using both the full spectra and spectra trimmed to match the smaller wavelength range.

We find that, regardless of subtype or which of the two wavelength ranges is used, SNID capably classifies events in the presence of strong reddening $(E(B-V) \sim$ $2.0 \mathrm{mag}$ and $R_{V}=3.1$ ), so long as the spectra exhibit a $\mathrm{S} / \mathrm{N} \gtrsim 1-3$. This is to be expected, as SNID divides input spectra by a pseudo-continuum fit and discards the spectral color information before performing crosscorrelation (Blondin \& Tonry 2007). In contrast, classifications performed via visual comparison may be prone to error when strong reddening is present.

At moderate and high noise levels $(\mathrm{S} / \mathrm{N} \lesssim 3)$, the post-maximum spectrum of SN Ic 2003aa could be con- 
fused with a SN Ib spectrum while the near-maximumlight spectrum is still identified as that of a SN Ic (the $\mathrm{He} \mathrm{I}$ lines in $\mathrm{SNe} \mathrm{Ib}$ fade as the event nears the nebular phase; e.g., Modjaz et al. 2014). At extremely high noise levels $(\mathrm{S} / \mathrm{N} \lesssim 1)$ SNID prefers a SN Ic-BL classification for SN 2003aa, especially when examining the post-maximum spectrum or using spectra covering only the smaller wavelength range, which does not capture the strong Ca II near-infrared (IR) triplet feature.

We also find that, if the SN redshift is uncertain and the $\mathrm{S} / \mathrm{N}$ is low, SN Ic spectra can be confused with SN Ia spectra (as shown by Blondin \& Tonry 2007), and so incorporating an independently measured redshift is helpful. Though SN 2004aw is not in the sample discussed here, it offers a nice illustration of the sometimesconfounding similarities between spectra of Type Ia and Type Ic SNe (e.g., Matheson et al. 2004a; Benetti et al. 2004; Filippenko et al. 2004).

Examining SN 1998dt, we find that SNID correctly identifies it as a $\mathrm{SN}$ Ib at both phases using spectra with $\mathrm{S} / \mathrm{N} \gtrsim 1$, and though the classification becomes very uncertain using only spectra with $\mathrm{S} / \mathrm{N} \lesssim 1$ and a restricted wavelength range, SNID never prefers an incorrect label.

SN 2003ed was correctly identified as a Type IIb SN so long as we examined spectra with $\mathrm{S} / \mathrm{N} \gtrsim 1$. Unlike
SN 1998dt, however, including spectra covering an extended wavelength range did not significantly affect the results even at low $\mathrm{S} / \mathrm{N}$, since the $\mathrm{He} \mathrm{I} \lambda 5876$ and $\mathrm{H} \alpha$ features proved the most useful and they are captured by all of our spectra.

Based on the above discussion, we adopt the following guidelines to avoid systematic biases when using SNID for our stripped-envelope classifications. First, it is difficult to discriminate between $\mathrm{SNe} \mathrm{Ib}$ and Ic or between $\mathrm{SNe} \mathrm{IIb}$ and Ib if only noisy $(\mathrm{S} / \mathrm{N} \lesssim 3)$ spectra observed at more than a few weeks post-maximum are available. Second, SNe Ic can be mislabeled as SNe Ic-BL when only low-S/N spectra are available.

\section{UPDATED CLASSIFICATIONS}

For the bulk of the SNe in the sample, especially the SNe Ia, our methods robustly confirm the L11 classifications. ${ }^{13}$ Table 1 lists all SNe in this sample, the type and subtype labels used by L11, and our updated labels. In the ensuing subsections, we discuss each changed classification individually as well as the rare subtypes and the uncertain and peculiar events we find. Many of the comparison spectra used in this section were drawn from the updated SNID template set; citations to the original publications are given.

Table 1. Updated Classifications of SNe in the LOSS Volume-Limited Sample

\begin{tabular}{|c|c|c|c|c|c|c|c|}
\hline Name & Previous (L11) & This Work & Ref. & Name & Previous (L11) & This Work & Ref. \\
\hline SN 1998de & Ia-91bg & Ia-91bg & $1,2,2$ & SN 1998dh & Ia-norm & Ia-norm & 2,3 \\
\hline SN 1998dk & Ia-norm & Ia-norm & 2,3 & SN 1998dm & Ia-norm & Ia-norm & 2,3 \\
\hline SN 1998dt & $\mathrm{Ib}$ & $\mathrm{Ib}$ & 4 & SN 1998ef & Ia-norm & Ia-norm & 3 \\
\hline SN $1998 \mathrm{es}^{1}$ & Ia-91T & Ia-99aa & 2,3 & SN 1999D & IIP & II & 5 \\
\hline SN 1999aa ${ }^{1}$ & Ia-91T & Ia-99aa & $2,3,6$ & SN $1999 \mathrm{ac}^{1}$ & Ia-91T & Ia-99aa/Ia-norm & $2,3,7$ \\
\hline SN 1999an & IIP & II & - & SN 1999bg & IIP & II & 5 \\
\hline SN 1999bh ${ }^{1}$ & Ia- $02 \mathrm{cx}$ & Ia-02es & 8,9 & SN 1999br & IIP & II & - \\
\hline SN 1999bu & Ic & Ic & - & SN 1999bw & impostor & impostor & 10 \\
\hline SN 1999by & Ia-91bg & Ia-91bg & 3 & SN 1999cd & $\mathrm{IIb}$ & $\mathrm{IIb}$ & - \\
\hline SN 1999cl & Ia-norm & Ia-norm & 2,3 & SN $1999 \mathrm{cp}$ & Ia-norm & Ia-norm & 3 \\
\hline SN 1999da & Ia-91bg & Ia-91bg & 3 & SN $1999 \mathrm{dk}$ & Ia-norm & Ia-norm & 3 \\
\hline SN 1999dn & $\mathrm{Ib}$ & $\mathrm{Ib}$ & 4 & SN $1999 \mathrm{dq}^{1}$ & Ia-91T & Ia-99aa & 2,3 \\
\hline SN 1999ej & Ia-norm & Ia-norm & 2 & SN 1999ek & Ia-norm & Ia-norm & 3 \\
\hline SN 1999el & IIn & IIn & - & SN $1999 \mathrm{em}$ & IIP & II & 5,11 \\
\hline SN 1999gd & Ia-norm & Ia-norm & 2,3 & SN 1999gi & IIP & II & 12 \\
\hline SN 1999go & IIL & II & 13 & $\mathrm{SN} 2000 \mathrm{C}$ & Ic & Ic & - \\
\hline $\mathrm{SN} 2000 \mathrm{H}$ & IIb & IIb & 14 & SN 2000L & IIP & II & - \\
\hline SN $2000 N$ & IIb/IIL & II & - & $\mathrm{SN} 2000 \mathrm{cb}^{2}$ & IIP & II- $87 \mathrm{~A}$ & 15 \\
\hline SN 2000ch & impostor & impostor & 10,16 & SN $2000 d c$ & IIL & II & 17 \\
\hline SN $2000 \mathrm{dm}$ & Ia-norm & Ia-norm & 3 & SN $2000 \mathrm{dr}$ & Ia-norm & Ia-norm & 3 \\
\hline
\end{tabular}

13 Note that, in a small number of cases, L11 reclassified some events from the original type announced in the CBETs; in this article we only discuss differences relative to the L11 labels. 
Table 1 (continued)

\begin{tabular}{|c|c|c|c|c|c|c|c|}
\hline Name & Previous (L11) & This Work & Ref. & Name & Previous (L11) & This Work & Ref. \\
\hline SN 2000el & IIP & II & - & SN 2000eo & IIn & IIn & - \\
\hline SN 2000ex & IIP & II & - & SN 2001E & Ia-norm & Ia-norm & 3 \\
\hline SN 2001J & IIP & II/IIb & - & SN $2001 \mathrm{~K}$ & IIP & II & - \\
\hline SN 2001L & Ia-norm & Ia-norm & 3 & SN 2001M & Ic & Ib & - \\
\hline SN 2001Q & $\mathrm{IIb}$ & $\mathrm{IIb}$ & - & SN $2001 V^{1}$ & Ia-91T & Ia-99aa & 2,3 \\
\hline SN 2001ac & impostor & impostor & 10 & SN 2001bq & IIP/IIL & II & 5 \\
\hline SN 2001ci & Ic & Ib & - & SN $2001 \mathrm{~cm}$ & IIP & II & 5,18 \\
\hline SN 2001dc & IIP & II & 19 & SN 2001dn & Ia-norm & Ia-norm & 3 \\
\hline SN 2001do & IIL & II & 17 & SN 2001en & Ia-norm & Ia-norm & 3 \\
\hline SN 2001ep & Ia-norm & Ia-norm & 3,20 & SN 2001fh & Ia-norm & Ia-norm & 3 \\
\hline SN $2001 \mathrm{fz}$ & IIP & II & - & SN 2001hf & IIL & II & - \\
\hline SN 2001is & $\mathrm{Ib}$ & $\mathrm{Ib}$ & - & $\mathrm{SN} 2002 \mathrm{~J}$ & Ic & Ic & - \\
\hline SN 2002an & IIL & II & 5 & SN 2002ap & Ic-pec & Ic-BL & 21 \\
\hline SN 2002bo & Ia-norm & Ia-norm & 3 & $\mathrm{SN} 2002 \mathrm{bu}^{3}$ & IIn & impostor & 10 \\
\hline SN 2002bx & IIP & II & 5 & SN 2002ca & IIP & II & 5 \\
\hline SN 2002ce & IIP & II & - & SN 2002cf & Ia-91bg & Ia-91bg & 3 \\
\hline SN 2002cr & Ia-norm & Ia-norm & 3 & SN 2002dj & Ia-norm & Ia-norm & 3 \\
\hline SN 2002dk & Ia-91bg & Ia-91bg & 3 & SN 2002do & Ia-norm & Ia-norm & 3 \\
\hline SN 2002dq & IIP & II & - & $\mathrm{SN} 2002 \mathrm{ds}^{4}$ & IIP & II & - \\
\hline SN 2002er & Ia-norm & Ia-norm & 3 & SN 2002es ${ }^{1}$ & Ia- $02 \mathrm{cx}$ & Ia-02es & 22 \\
\hline $\mathrm{SN} 2002 \mathrm{fb}$ & Ia-91bg & Ia-91bg & 3 & SN $2002 \mathrm{fk}$ & Ia-norm & Ia-norm & 3 \\
\hline SN 2002gd & IIP & II & 5 & SN 2002gw & IIP & II & - \\
\hline SN 2002ha & Ia-norm & Ia-norm & 3 & SN $2002 \mathrm{hh}$ & IIP & II & 5 \\
\hline SN 2002hw & Ia-norm & Ia-norm & 3 & SN 2002jg & Ia-norm & Ia-norm & 3 \\
\hline SN 2002jj & Ic & Ic/Ic-BL & - & SN 2002jm & Ia-91bg & Ia-91bg & 3 \\
\hline SN 2002jz & Ic & $\mathrm{Ib} / \mathrm{Ic}$ & - & SN $2002 \mathrm{~kg}$ & impostor & impostor & 23,24 \\
\hline SN 2003E & IIP & II & - & SN 2003F & Ia-norm & Ia-norm & 3 \\
\hline SN 2003G & IIn & IIn & - & $\mathrm{SN} 2003 \mathrm{H}^{5}$ & Ibc-pec & Ca-rich & 25 \\
\hline SN 2003Y & Ia-91bg & Ia-91bg & 3 & SN $2003 Z$ & IIP & II & 5 \\
\hline SN 2003aa & Ic & Ic & - & SN 2003ao & IIP & II & - \\
\hline SN 2003bk & IIP & II & - & $\mathrm{SN} 2003 \mathrm{br}$ & IIP & II/IIb & - \\
\hline SN 2003bw ${ }^{4}$ & IIP & II/IIb & - & SN $2003 \mathrm{cg}$ & Ia-norm & Ia-norm & 26 \\
\hline $\mathrm{SN} 2003 \mathrm{dr}^{5}$ & Ibc-pec & Ca-rich & 25 & SN 2003du & Ia-norm & Ia-norm & 3,27 \\
\hline SN 2003dv & IIn & IIn & 28 & SN 2003ed & $\mathrm{IIb}$ & $\mathrm{IIb}$ & - \\
\hline SN 2003ef & IIP & II & - & SN 2003gm & impostor & impostor & 23 \\
\hline SN 2003gt & Ia-norm & Ia-norm & 3 & SN 2003hg & IIP & II & 13 \\
\hline $\mathrm{SN} 2003 \mathrm{hl}$ & IIP & II & 5 & SN 2003id & Ic-pec & Ic-pec & - \\
\hline SN 2003iq & IIP & II & 5 & SN 2003kf & Ia-norm & Ia-norm & 3 \\
\hline SN 2003ld & IIP & II & - & SN 2004C & Ic & IIb & - \\
\hline SN 2004ab & Ia-norm & Ia-norm & 9 & SN 2004al & IIb/IIL & II & - \\
\hline SN 2004aq & IIP & II & - & SN 2004bd & Ia-norm & Ia-norm & 3 \\
\hline SN 2004be & $\mathrm{IIb}$ & $\mathrm{IIb}$ & - & SN 2004bl & Ia-norm & Ia-norm & 3 \\
\hline SN 2004bm & Ibc-pec/IIb & IIb/IIb-pec & - & SN 2004bv & Ia-91T & Ia-91T & 3 \\
\hline SN 2004ca & Ia-norm & Ia-norm & 3 & SN 2004cc & Ic & $\mathrm{Ib} / \mathrm{Ic}$ & 13 \\
\hline SN 2004ci & IIP & II & - & SN 2004dd & IIP & II & - \\
\hline SN $2004 \mathrm{dk}$ & $\mathrm{Ib}$ & $\mathrm{Ib}$ & - & SN 2004er & IIP & II & - \\
\hline SN 2004et & IIP & II & 5 & SN 2004fc & IIP & II & - \\
\hline SN 2004fx & IIP & II & 29 & SN 2004gq & $\mathrm{Ib}$ & $\mathrm{Ib}$ & 14 \\
\hline SN 2005A & Ia-norm & Ia-norm & 3,30 & SN $2005 \mathrm{E}^{5}$ & Ibc-pec & Ca-rich & 25,31 \\
\hline SN $2005 \mathrm{H}$ & IIb & II/IIb & 13 & SN 2005J & IIL & II & - \\
\hline SN $2005 \mathrm{U}$ & $\mathrm{IIb}$ & $\mathrm{IIb}$ & 14 & SN $2005 \mathrm{~W}$ & Ia-norm & Ia-norm & 3,30 \\
\hline
\end{tabular}


Table 1 (continued)

\begin{tabular}{|c|c|c|c|c|c|c|c|}
\hline Name & Previous (L11) & This Work & Ref. & Name & Previous (L11) & This Work & Ref. \\
\hline SN 2005ad & IIP & II & - & SN 2005am & Ia-norm & Ia-norm & 3,30 \\
\hline SN 2005an & IIL & II & - & SN 2005aq & IIn & IIn & - \\
\hline SN 2005as & Ia-norm & Ia-norm & 3 & SN 2005ay & IIP & II & 5 \\
\hline SN 2005az & Ic & Ic & 14 & SN 2005bb & IIP & II & - \\
\hline SN 2005bc & Ia-norm & Ia-norm & 3 & SN 2005bo & Ia-norm & Ia-norm & 3,30 \\
\hline SN 2005cc & Ia- $02 \mathrm{cx}$ & Ia- $02 \mathrm{cx}$ & 9 & SN $2005 \mathrm{cf}$ & Ia-norm & Ia-norm & 32 \\
\hline SN $2005 \mathrm{ci}^{2}$ & IIP & II- $87 \mathrm{~A}$ & 15 & SN 2005de & Ia-norm & Ia-norm & 3 \\
\hline SN 2005el & Ia-norm & Ia-norm & 3,30 & SN $2005 \mathrm{hk}$ & Ia- $02 c x$ & Ia- $02 c x$ & $3,33,34$ \\
\hline SN 2005io ${ }^{2}$ & IIP & II-87 A & - & SN $2005 \mathrm{kc}$ & Ia-norm & Ia-norm & 3,30 \\
\hline SN 2005ke & Ia-91bg & Ia-91bg & 3,30 & SN 2005lr & Ic & IIb & - \\
\hline SN $2005 \mathrm{mg}$ & IIP & II/IIb & - & SN $2005 \mathrm{mz}$ & Ia-91bg & Ia-91bg & 9 \\
\hline SN $2006 \mathrm{~F}$ & $\mathrm{Ib}$ & $\mathrm{Ib}$ & - & SN 2006T & $\mathrm{IIb}$ & $\mathrm{IIb}$ & - \\
\hline SN 2006X & Ia-norm & Ia-norm & 3,30 & SN 2006ax & Ia-norm & Ia-norm & 3,30 \\
\hline SN 2006be & IIP & II & - & SN 2006bp & IIP & II & - \\
\hline $\mathrm{SN} 2006 \mathrm{bv}^{3,4}$ & IIn & impostor & 10 & SN 2006ca & IIP & II & - \\
\hline SN $2006 \mathrm{~cm}^{1}$ & Ia-91T & Ia-99aa/Ia-norm & 3 & SN 2006dy & Ia-norm & Ia-norm & 3 \\
\hline SN $2006 \mathrm{ef}$ & Ia-norm & Ia-norm & 3,30 & SN 2006eg & Ic & $\mathrm{IIb} / \mathrm{Ib} / \mathrm{Ic} / \mathrm{Ic}-\mathrm{BL}$ & - \\
\hline SN 2006ke & Ia-91bg & Ia-91bg & 3 & SN 2006le & Ia-norm & Ia-norm & 3 \\
\hline SN 2006lf & Ia-norm & Ia-norm & 3 & SN 2006qr & IIP & II & - \\
\hline
\end{tabular}

This table lists the previous classifications for all objects in L11 and our confirmed or updated classifications. Notable objects, discussed individually within the text, are printed in boldface. We also list references to the original publishers for all data already in the literature that were used in this effort. When we cannot confim a single clear classification, we list more than one possible type or subtype. See $\S 3$ for a detailed description of each type and subtype label.

References: [1] Modjaz et al. (2001); [2] Matheson et al. (2008); [3] Silverman et al. (2012); [4] Matheson et al. (2001); [5] Faran et al. (2014b); 6] Garavini et al. (2004); [7] Garavini et al. (2005); [8] Li et al. (2001); [9] Blondin et al. (2012); [10] Smith et al. (2011b); [11] Leonard et al. (2002b); [12] Leonard et al. (2002a); [13] Harutyunyan et al. (2008); [14] Modjaz et al. (2014); [15] Kleiser et al. (2011); [16] Wagner et al. (2004); [17] Faran et al. (2014a); [18] Poznanski et al. (2009); [19] Pastorello et al. (2004); [20] Sauer et al. (2008); [21] Foley et al. (2003); [22] Ganeshalingam et al. (2012); [23] Maund et al. (2006); [24] Van Dyk et al. (2006); [25] Perets et al. (2010); [26] Elias-Rosa et al. (2006); [27] Leonard et al. (2005); [28] Bilinski et al. (2015); [29] Hamuy et al. (2006); [30] Folatelli et al. (2013); [31] Foley et al. (2009); [32] Wang et al. (2009b); [33] Chornock et al. (2006); [34] Phillips et al. (2007)

1 See $\S 4.3$

2 See $\S 4.5$

3 See $\$ 4.7$

${ }^{4}$ See $\S 4.8$

5 See $\S 4.6$

\subsection{Reclassified Objects}

4.1.1. $S N$ 2000N $(I I b / I I L \rightarrow I I)$

SN 2000N was discovered in MCG-02-34-054 (Sato et al. 2000) and classified as a Type II SN from a spectrum with a low S/N (Jha et al. 2000). However, because the data they had on this object were quite sparse, L11 could not determine if SN 2000N was a Type IIb or a Type II SN. We were able to obtain the spectrum originally used to classify the SN as well as additional spectra, including one near peak brightness when the spectra of SNe IIb and II are more clearly differentiable; see Figure ??. SNID identifies SN 2000N as a Type II SN, and our rereduced light curve indicates that the SN IIL template from L11 is a better match than the SN IIb one.

\subsection{2. $S N$ 2001M $(I c \rightarrow I b)$}

SN 2001M was discovered in NGC 3240 and classified as a SN Ic (Aazami \& Li 2001; Suntzeff et al. 2001; Filippenko et al. 2001a). SNID identifies reasonably good cross-correlations with spectra of both $\mathrm{SNe} \mathrm{Ib}$ and SNe Ic. We have been able to locate only a single spectrum of this object and our light curve is sparse, so the phase of our spectrum is somewhat uncertain; see Figure ??. Narrow, unresolved $\mathrm{H} \alpha$ emission from the star-forming host galaxy partially obscures the He I $\lambda 6678$ absorption line, but we do identify probable weak He I $\lambda 6678$ and He I $\lambda 7065$ lines at $v \approx 8500 \mathrm{~km} \mathrm{~s}^{-1}$. The region around He I $\lambda 5876$ is complex, with what must be several overlapping absorption lines; we believe it to be consistent with He I $\lambda 5876$ ab- 

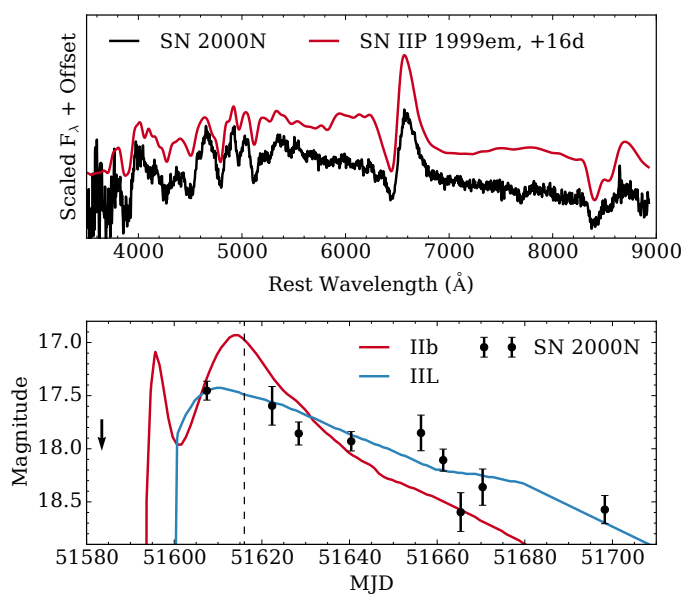

Figure 2. Top: the near-maximum-light spectrum of SN 2000N alongside that of the Type IIP SN 1999em (Leonard et al. 2002b). Bottom: the light curve of SN 2000N, with a vertical line showing the date the spectrum shown was observed, and colored lines showing the template light curves from L11.

sorption but not dominated by it.

We compare our spectrum to the +10 day average SN Ib and SN Ic spectra of Liu et al. (2016), after estimating a date of peak of 24 January 2001 from the light curve (implying a phase of $+7.5 \mathrm{~d}$ for our spectrum). This comparison shows many similarities between SN 2001M and both classes, but reinforces our identifications of the He I lines. Given our detections of both He I $\lambda 6678$ and He I $\lambda 7065$, we relabel SN $2001 \mathrm{M}$ as a Type Ib SN, though we note that the helium lines are weak compared to those in most $\mathrm{SNe} \mathrm{Ib}$.

\subsection{3. $S N$ 2001ci $(I c \rightarrow I b)$}

SN 2001ci was discovered in NGC 3079 (Swift et al. 2001) and announced as a SN Ic heavily obscured by host-galaxy dust absorption (Filippenko \& Chornock 2001). A re-examination of the spectrum cited therein (observed UT 2001-05-30; we were not able to locate any other spectra of this object) confirms that it is heavily reddened by host-galaxy dust. The MW contribution to the reddening is only $E(B-V)=0.0097 \mathrm{mag}$ (Schlafly \& Finkbeiner 2011, used for all subsequent MW reddening measures), but the spectrum clearly shows narrow Na I absorption features from the host galaxy indicating significant reddening produced by host-galaxy dust. The spectrum is of sufficiently high resolution to resolve both components of the Na I D doublet, but their equivalent widths $(\mathrm{EWs})$ are well outside the range of the empirical relations of Poznanski et al. (2012): $E W_{D 1} \approx 2.4 \AA$ and $E W_{D 2} \approx 2.2 \AA$. This implies that $E(B-V) \gtrsim 3.0$ mag.

Note that SNID, by construction, is insensitive to color information and to uncertainties in the reddening corrections or flux calibrations (Blondin \& Tonry 2007). Throughout this paper we apply (often uncertain) red-

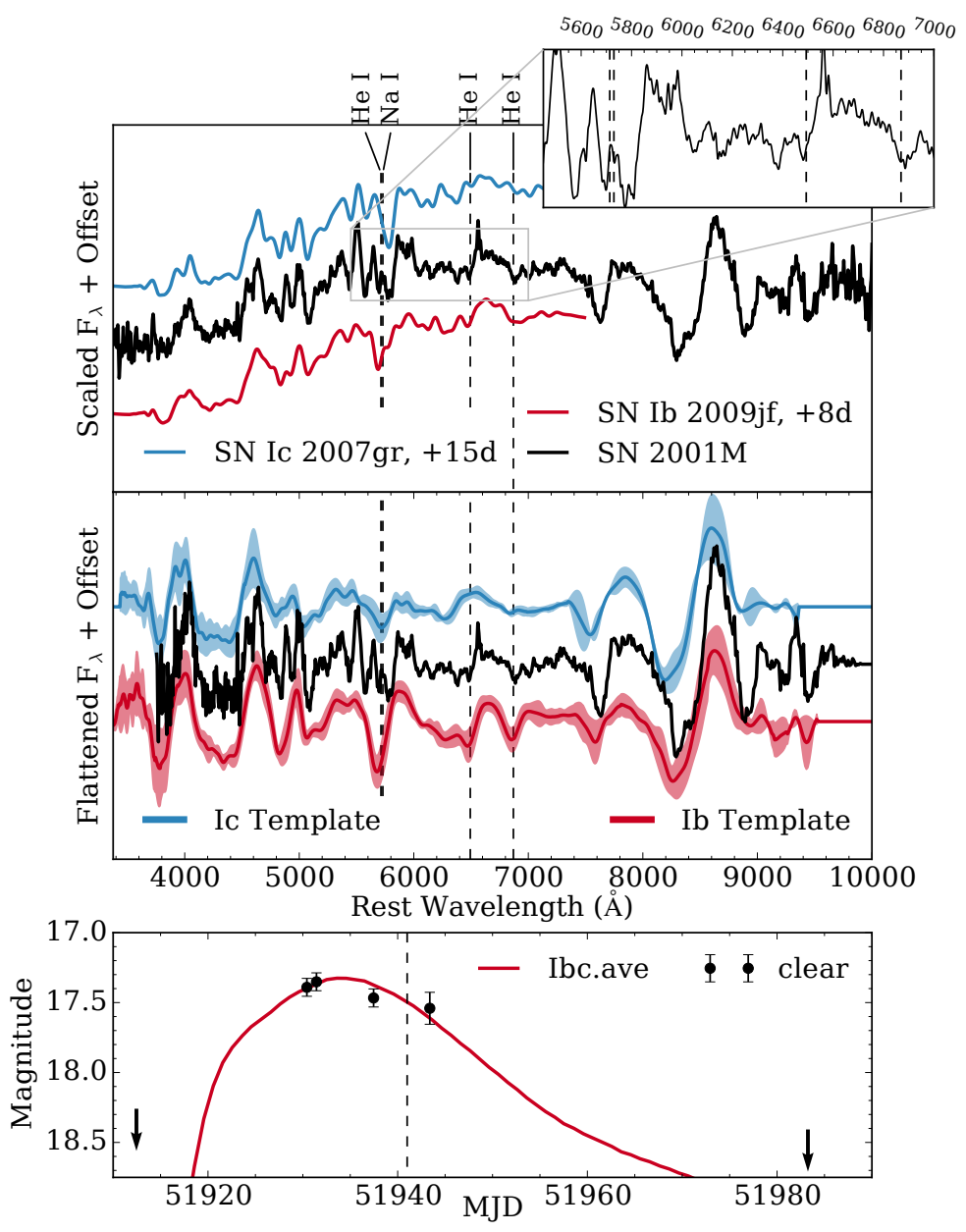

Figure 3. Top: spectrum of SN 2001M smoothed with a $20 \AA$ Gaussian kernel, and with weak helium features identified. We also show spectra of SN Ic $2007 \mathrm{gr}$ and SN Ib 2009jf (Modjaz et al. 2014), and the wavelengths of He I and Na I features are marked at $v=8300 \mathrm{~km} \mathrm{~s}^{-1}$. The inset shows the He I lines we identify in the SN 2001M. Middle: the continuum-normalized spectrum of SN 2001M compared to the $+10 \mathrm{~d}$ average spectra of $\mathrm{Ib}$ and Ic SNe from Liu et al. (2016), with the standard deviation shown as shaded regions. Bottom: light curve of SN 2001M compared to the average $\mathrm{Ib} / \mathrm{c}$ template light curve from L11, with the date of spectrum shown with a dashed line.

dening corrections to spectra to facilitate visual comparisons, but they do not appreciably affect the SNID classifications.

Adopting $E(B-V)=2.0 \mathrm{mag}$ and a MW-like dust law is good enough to achieve a robust identification: SN 2001ci is a Type Ib SN, with a spectrum that is most similar to those of SNe Ib with weak helium lines. See Figure 4 for a comparison to the He-weak SN Ib 1999ex (Hamuy et al. 2002). Filippenko \& Chornock (2001) drew attention to a similarity with SN 1990U, which was (at the time) classified as a SN Ic, but Modjaz et al. (2014) have subsequently reclassified SN 1990U as a SN Ib. 

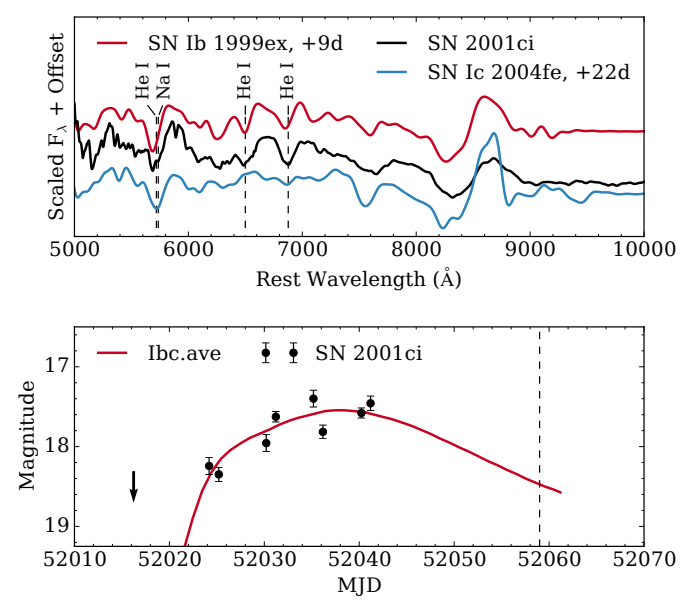

Figure 4. Top: spectrum of SN 2001ci (corrected for a reddening of $E(B-V)=3.0 \mathrm{mag}$ and smoothed with a Gaussian kernel $50 \AA$ wide), compared with that of the Type Ib SN 1999ex (Hamuy et al. 2002) and the Type Ic SN 2004fe (Modjaz et al. 2014). Bottom: the light curve compared to a L11 template with the date of the spectrum marked by a vertical line.

\subsection{4. $S N 2004 C(I c \rightarrow I I b)$}
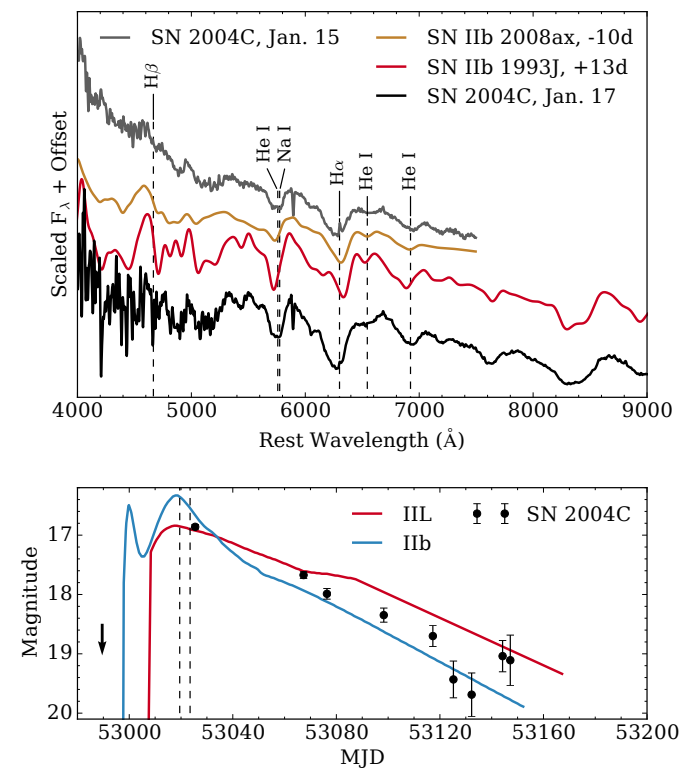

Figure 5. Top: spectra of SN 2004C observed on UT 200401-15 and on UT 2004-01-17, alongside a premaximum spectrum of the Type IIb SN 2008ax (Modjaz et al. 2014) and a post-maximum spectrum of the Type IIb SN 1993J (Matheson et al. 2000). Both spectra of SN 2004C have been dereddened by $E(B-V)=1.0 \mathrm{mag}$ and smoothed by a $20 \AA$ Gaussian kernel, and galaxy emission lines have been subtracted by hand. Bottom: light curve of SN 2004C compared to templates from L11 with the date of the spectra marked by vertical lines.

SN 2004C was discovered in NGC 3683 (Dudley \& Fischer 2004) and classified as a heavily reddened SN Ic
(Matheson et al. 2004b), spectrally similar to SN 1990U (see §4.1.3: SN 1990U was a SN Ib). The MW reddening along the line of sight is only $E(B-V)=$ $0.0133 \mathrm{mag}$, but (unresolved) Na I D absorption in our spectra indicates strong host-galaxy obscuration. As with SN 2001ci, the Na I D EW is well beyond the empirical relations of Poznanski et al. (2012), but the spectra do not appear to be as reddened as those of SN 2001ci. Correcting for a total reddening of $E(B-V)=1.0 \mathrm{mag}$ produces a reasonable result.

Here we publish several spectra of SN 2004C which indicate that SN 2004C was a Type IIb SN. Figure 5 shows a comparison between the spectrum announced by Matheson et al. (2004b) and the Type IIb SN 2008ax, as well as a later spectrum with broader wavelength coverage compared to a spectrum of the Type IIb SN 1993J.

\subsection{5. $S N$ 2004al $(I I b / I I L \rightarrow I I)$}

SN 2004al was discovered in ESO 565-G25 (Singer et al. 2004) and classified as a Type II SN (Matheson et al. 2004c). As L11 show, SN 2004al had a light curve consistent with either a SN IIb or a SN IIL classification, and so they assign it a $50 \%$ weight in each class. Although we have no additional spectra to examine, we were able to obtain the classification spectrum and SNID clearly identifies it as a Type II SN, not a SN IIb, mostly owing to the absence of He I $\lambda 5876$. Figure 6 shows that our spectrum was observed $\sim 1$ week postmaximum; though He I lines are often weak in young $\mathrm{SNe}$ IIb, they become pronounced by maximum light. In good agreement with L11, our rereduced light curve exhibits a relatively rapid decline and is better fit by the SN IIb template; see Figure 6. Despite this tension, we consider the spectroscopic classification robust and we label SN 2004al a Type II event.

\subsubsection{SN 2005io $(I I P \rightarrow I I-87 A)$}

SN 2005io was discovered in UGC 3361 (Lee et al. 2005) and reported as a young Type II SN (Filippenko \& Foley 2005) based upon a Keck LRIS spectrum. The photometry of SN 2005io shows a peculiar evolution, however - see Figure 7. The light curve initially follows the Type IIP template almost exactly, but after peaking at an absolute magnitude of $-15.2 \mathrm{mag}$ SN 2005io goes into a slight decline and then a very slow rise (over $\sim 100$ days $)$ to a second maximum $(<-15.75 \mathrm{mag}$; the peak itself went unobserved). This is similar to the photometric behavior of SN 1987A and other related events, though in SN 1987A the early peak was only visible in bluer passbands and the rise to its second, more luminous, peak occurred more rapidly. The spectrum is similar to that of a normal young Type II SN with hydrogen P-Cygni profiles dominated by the emission component and with an absorption Doppler velocity of 

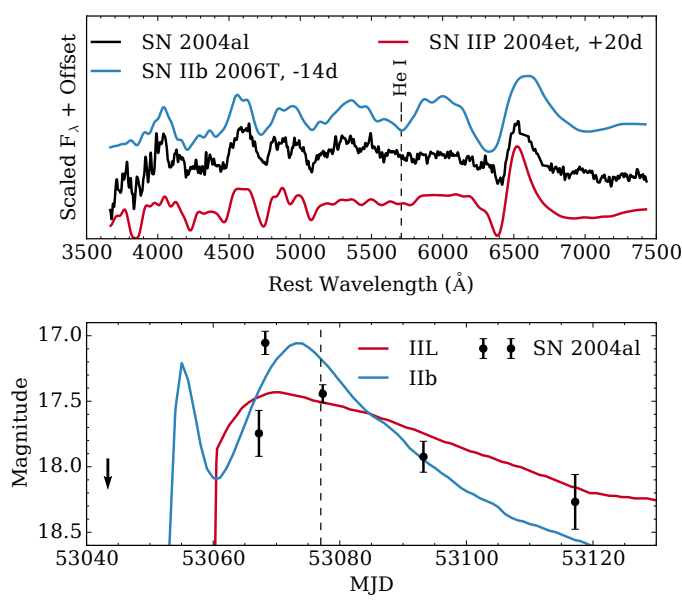

Figure 6. Top: spectrum of SN 2004al smoothed with a $20 \AA$ Gaussian kernel and compared to spectra of the Type IIP SN 2004et a few weeks after maximum (Sahu et al. 2006) and the premaximum SN IIb 2006T (the top non-IIP SNID template, Modjaz et al. 2014). Bottom: the light curve compared to templates from L11 with the date of the spectrum marked by a vertical line.

$\sim 8,000 \mathrm{~km} \mathrm{~s}^{-1}$, slower than the velocity found for hydrogen in SN 1987A.

Based primarily upon the light-curve evolution, and noting that the subclass is heterogeneous (Pastorello et al. 2005b; Kleiser et al. 2011; Taddia et al. 2012; Pastorello et al. 2012; Taddia et al. 2016b), we classify SN 2005io as a SN 1987A-like event alongside the two other SN 1987A-like events already identified within the L11 sample (SNe 2000cb and 2005ci; Kleiser et al. 2011). It unfortunately appears that the peculiarity of SN 2005io was not recognized while it was bright: we believe that Figure 7, which shows the classification spectrum and the KAIT photometry, includes all extant observations of the event. Though the central wavelength of unfiltered KAIT photometry is quite similar to that of the $R$ band, the effective passband is significantly wider (Ganeshalingam et al. 2010). Our early-time spectrum of SN 2005io shows that it was quite blue while young, and it's likely that the rapid rise to the first photometric peak was powered by cooling envelope emission, as was observed in SN 1987A via photometry in bluer passbands.

The second peak of SN 2005io lasts longer than that of SN 1987A, and the fade from peak was not observed. We have upper limits showing that the object had faded to $\gtrsim 19.7 \mathrm{mag}$ (absolute mag -14.2 ) by Sep. 18 , but we have no data obtained between the last detection on Feb. 24 (shown in Figure 7) and then. SN 2005io shares some similarities with SN 2009E (Pastorello et al. 2012), including a slow rise and faint peak compared to the prototypical SN 1987A. The early peak and slow rise to second maximum is also reminiscent of SN 2004ek
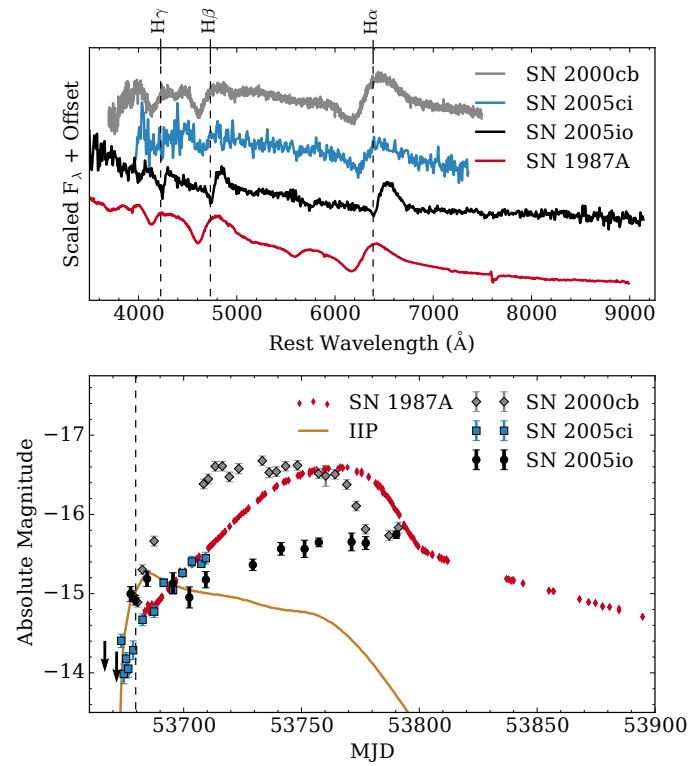

Figure 7. Top: spectrum of SN 2005io compared to spectra of the young SNe 2000cb, 2005ci, and 1987A (SNe 2000cb and 2005ci are also included in the LOSS volume-limited sample; Blanco et al. 1987; Kleiser et al. 2011). The spectra of SNe 2005io and 2005ci have been smoothed by a Gaussian kernel $20 \AA$ wide. We mark prominent hydrogen features at $8000 \mathrm{~km} \mathrm{~s}^{-1}$. Bottom: light curve of SN 2005 io (with a vertical line showing the date the spectrum was observed) compared to the light curves of the other SN 1987A-like events after correcting for distance and MW dust absorption along the line of sight, and then offset in time to align their initial rises (Hamuy \& Suntzeff 1990; L11). Also shown is the template Type IIP light curve from L11, offset to match the early evolution of SN 2005io.

(Taddia et al. 2016b), although that SN was a great deal more luminous $(R \approx-18.5 \mathrm{mag})$ than SN 2005io (clear $\lesssim-15.75 \mathrm{mag})$, further illustrating the heterogenous nature of these slow-rising SNe II.

\subsection{7. $S N$ 2005lr $(I c \rightarrow I I b)$}

SN 2005lr was discovered in ESO 492-G2 (Baek \& Li 2005) and was spectroscopically classified as a SN Ic, with spectra similar to those of SN 1990B (Hamuy et al. 2005). We obtained a copy of the classification spectrum for SN 2005lr from the CSP archives (observed 2005 Dec. 18 ) as well as a second (higher $\mathrm{S} / \mathrm{N}$ ) spectrum taken two days later.

The spectra of SN 2005lr show that this SN was strongly reddened by host-galaxy dust - the Na ID line in these low-resolution spectra exhibits a total EW of $\sim 2.4 \AA$, just beyond the limits of the relations from Poznanski et al. (2012). We again deredden the spectrum by an arbitrary value of $E(B-V)=1.0$ mag to facilitate visual inspection.

SNID identifies SN 2005lr as either a SN Ib or IIb, with matches to examples of either class. Distinct He I, O I, and $\mathrm{Ca}$ II lines are detected alongside an $\mathrm{H} \alpha$ absorption 

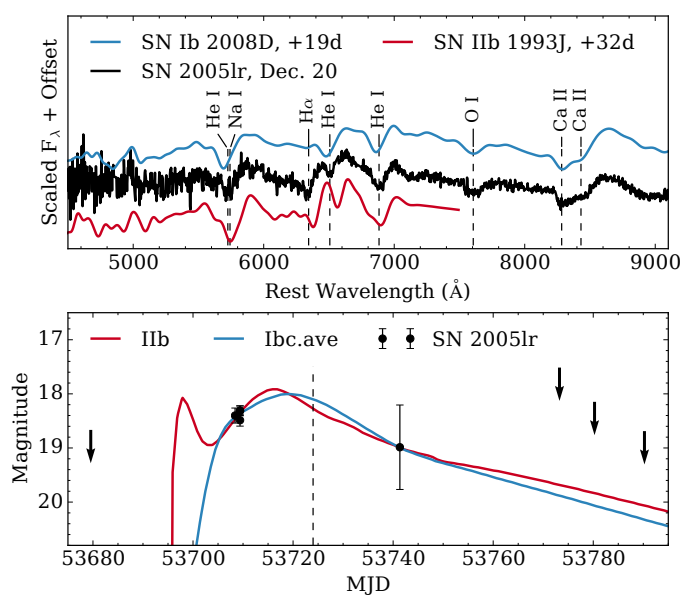

Figure 8. Top: the higher S/N spectrum of SN 2005lr smoothed with a Gaussian kernel $10 \AA$ wide and compared to spectra of the SN Ib 2008D and SN IIb 1993J (Matheson et al. 2000; Modjaz et al. 2009). Bottom: light curve of the SN compared to templates from L11 with date of spectrum shown by a vertical line.

feature; see Figure 8. Our spectra appear to be taken well after peak brightness (although the light curve is extremely sparse) and the detection of a relatively strong $\mathrm{H} \alpha$ feature at this phase identifies SN 2005lr as a Type IIb SN.

\subsection{Low-Certainty Classifications and Peculiar Events}

We have made an effort to track down spectra of every object in the volume-limited sample, especially those spectra originally used for classification in the CBETs, and re-examine the classifications of each. Unfortunately, however, there are several objects in our sample for which robust classifications are simply not possible given the peculiarity of the object or the quality of the data.

\subsection{1. $\quad S N$ 2001J $(I I P \rightarrow I I / I I b)$}

SN 2001J was discovered in UGC 4729 (Beckmann \& Li 2001) and classified as a Type II SN (Jha et al. 2001). SNID identifies the SN 2001J spectrum as that of a young SN IIb, similar to the spectrum of SN 2008ax. L11 list SN 2001J as a SN IIP with poor light-curve coverage, but Figure 9 shows our rereduced KAIT light curve (including nondetection upper limits) indicating there was no bright hydrogen recombination plateau phase. Taking into account both the SNID result and the rapid light-curve decay, we prefer the SN Type IIb classification, but cannot rule out the possibility that SN 2001J was a hydrogen-rich Type II SN with a relatively rapid decline rate.

\subsubsection{SN 2002jj $(I c \rightarrow I c / I c-B L)$}

SN 2002jj was discovered in IC 340 (Hutchings \& Li 2002) and classified as a SN Ic (Foley \& Filippenko
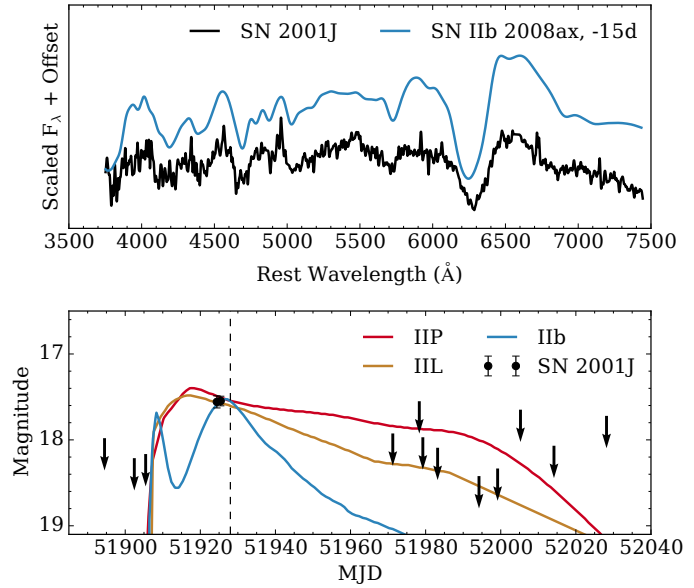

Figure 9. Top: the spectrum of SN 2001J (smoothed by a $20 \AA$ Gaussian kernel) compared to that of the Type IIb SN 2008ax (Modjaz et al. 2014). Bottom: the light curve compared to the Type IIL, IIP, and IIb templates from L11. Upper limits from nondetections are shown as arrows, and the date of the spectrum is marked with a dashed vertical line.

2002), with a spectrum similar to that of SN 1994I. We have three spectra of SN 2002jj, all of moderate quality. SNID prefers a SN Ic-BL classification, as do comparisons with the average spectra of Liu et al. (2016), but the data are not good enough to be sure. The light curve (though it is sparse) indicates that all of our spectra were taken well past peak brightness. SN 2002jj showed a peak absolute magnitude of $-17.66 \pm 0.23$ (L11), a value in the normal range for both SNe Ic and SNe Ic$\mathrm{BL}$ (SNe Ic-BL are, on average, more luminous than other stripped-envelope SNe; e.g., Drout et al. 2011; Lyman et al. 2016), and so it is not clear whether this SN was a bona-fide SN Ic-BL.

In addition, the $\mathrm{He}$ I lines used to distinguish $\mathrm{SNe}$ Ic from Ib are most apparent around the time of peak brightness and then fade, in most cases disappearing entirely by $\sim 50-70$ days (Modjaz et al. 2014; Liu et al. 2016). The spectroscopic coverage of SN 2002jj did not begin until $\sim 1$ month post-peak and we cannot rule out the possibility of weak helium features near peak, but we prefer the SN Ic or SN Ic-BL classification; see Figure 10.

\subsection{3. $S N$ 2002jz $(I c \rightarrow I b / I c)$}

SN 2002jz was discovered in UGC 2984 and classified as a SN Ic with a resemblance to SN 1994I (Puckett et al. 2002). We present three spectra of this object, the most useful of which was observed on UT 2003-01-07. There is significant MW dust reddening along the line of sight $(E(B-V)=0.4846 \mathrm{mag})$, and the unresolved Na I D absorption in our spectra indicates a similar amount of host-galaxy dust obscuration.

SNID weakly prefers a Type Ib label over the Ic label; the best SNID matches are to the spectra of the 

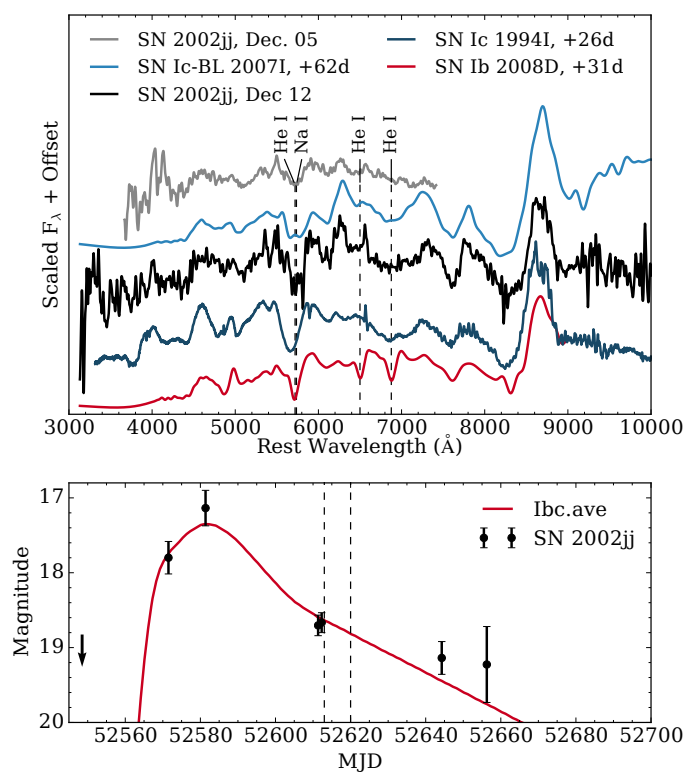

Figure 10. Top: spectra of SN 2002jj, smoothed by a $40 \AA$ Gaussian kernel and compared to spectra of the Type Ic-BL SN 2007I (Modjaz et al. 2014), the Type Ic SN 1994I (Filippenko et al. 1995), and the Type Ib SN 2008D (Modjaz et al. 2009). Bottom: light curve of SN 2002jj compared to the average $\mathrm{SN} \mathrm{Ib/c} \mathrm{template} \mathrm{from} \mathrm{L11,} \mathrm{with} \mathrm{the} \mathrm{dates} \mathrm{of}$ the spectra marked by vertical lines.

Type Ib SNe 1995F (reclassified from Ic to Ib by Modjaz et al. 2014) and 1999ex (studied in detail by, e.g., Parrent et al. 2007). However, the He I $\lambda 6678$ line in SN 2002jz is so weak as to be nearly indiscernible (it is only detected as a notch out of an adjacent P-Cygni emission profile), and the He I $\lambda 7065$ line is notably weaker than that of SN 1995F — see Figure 11. We give SN 2002jz equal weights in the Ib and Ic categories.

\subsection{4. $S N$ 2003br $(I I \rightarrow I I / I I b)$}

SN 2003br was discovered in MCG-05-34-18 (Swift \& Li 2003) and was classified as a Type II SN (Berlind et al. 2003). We present the classification spectrum of SN 2003br, but this alone is not enough to distinguish between a Type II or a Type IIb classification. The MW reddening toward the object is small $(E(B-V)=$ $0.0822 \mathrm{mag}$ ), but the observed spectral energy distribution (SED) implies that there must be a moderate-tolarge degree of host-galaxy reddening. Unfortunately, the spectrum has neither the signal nor the resolution to measure any possible narrow $\mathrm{Na} I \mathrm{D}$ absorption features. Adopting a correction of $E(B-V)=1.0 \mathrm{mag}$ appears to roughly correct the SED, and so we continue with that.

SNID indicates that the best match is with the Type IIb SN 2006T, but the Type IIP SN 1999em also shows a good match. The light curve is more similar to the Type IIL template, and so we prefer a Type II classification but cannot be sure. See Figure 12.
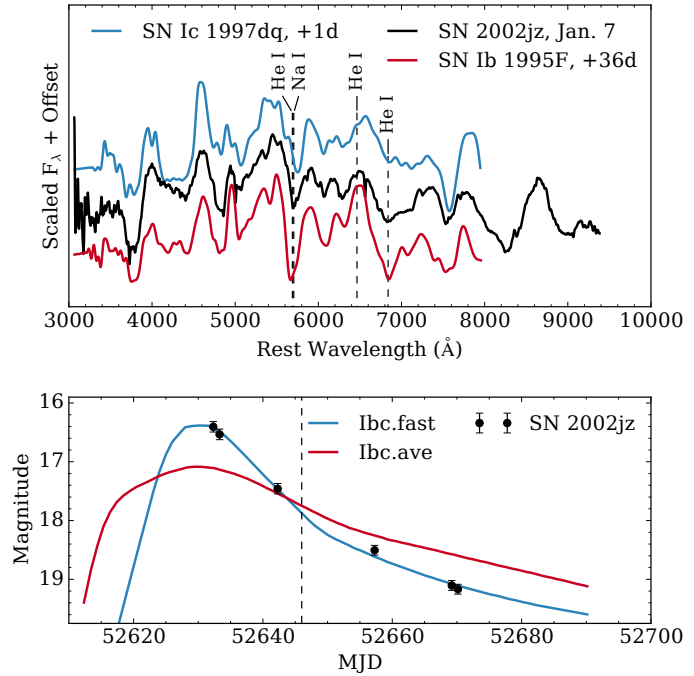

Figure 11. Top: spectrum of SN 2002jz from 2003-01-07 (corrected for a MW reddening of $E(B-V)=0.4846 \mathrm{mag}$ ) compared with that of SN Ic 1997dq and SN Ib 1995F (Matheson et al. 2001; Modjaz et al. 2014). Bottom: light curve of SN 2002jz compared to the stripped-envelope templates of L11, with the date of the spectrum shown with a vertical line.
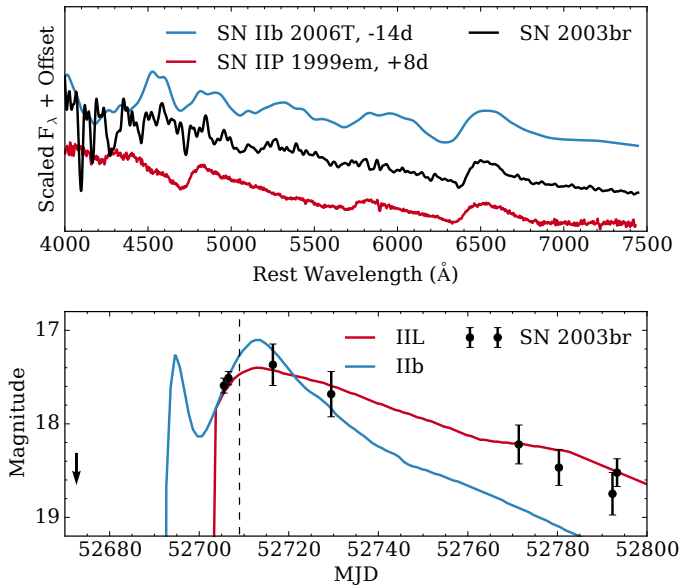

Figure 12. Top: spectrum of SN 2003br (corrected for $E(B-V)=1.0 \mathrm{mag}$ and smoothed with a $40 \AA$ Gaussian kernel), compared to that of the Type IIb SN 2006T (Modjaz et al. 2014) and the Type IIP SN 1999em (Leonard et al. 2002b) - both match well. Bottom: the light curve compared to the templates from L11, with the date of the spectrum marked.

\subsubsection{SN 2003id (Ic-pec)}

SN 2003id was discovered in NGC 895 and classified as a SN Ibc-pec by Hamuy \& Roth (2003), who identify several normal SN Ic lines as well as a strong blended feature around $5700 \AA$. Here we analyze spectra observed on Sep. 19 and Oct. 23, 2003 - see Figure 13. Our spectra confirm this to be an odd object with no good matches in the SNID template set, though with many 
similarities to the peculiar SN Ib 2007uy (Roy et al. 2013; Modjaz et al. 2014).

The strong blended feature around $5700 \AA$ persists and appears to grow stronger over time. We tentatively identify He I $\lambda 6678$ at $v \approx 11,000 \mathrm{~km} \mathrm{~s}^{-1}$, which implies that the feature at $5700 \AA$ may include some absorption from He I $\lambda 5876$ in addition to $\mathrm{Na} I \lambda \lambda 5890,5896$; perhaps this feature arises from multiple velocity components of these two ions. However, we find no clear sign of He I $\lambda 7065$ absorption in either spectrum. (Note that these spectra have undergone a correction for telluric absorption and none of the features in them is telluric.)

We also identify a feature near $6140 \AA$ that evolves from a strongly blended state into two clearly defined components, the redder of which is plausibly highvelocity $\mathrm{H} \alpha$ at $v \approx 17,500 \mathrm{~km} \mathrm{~s}^{-1}$, and we tentatively identify $\mathrm{H} \beta$ at $v \approx 17,500-18,500 \mathrm{~km} \mathrm{~s}^{-1}$ in the spectrum from Sep. 19. The bluer feature may be a second, even higher-velocity $\mathrm{H} \alpha$ line at $v \approx 23,500 \mathrm{~km} \mathrm{~s}^{-1}$; the bluemost edge of the Ca II IR triplet absorption implies similar velocities for the calcium in SN 2003id. Several authors have previously identified high-velocity $\mathrm{H} \alpha$ lines in SN Ib/c spectra (e.g., Branch et al. 2006; Elmhamdi et al. 2006; Parrent et al. 2007).

We compare the spectra of SN 2003id to the average spectra of Liu et al. (2016) in the middle panel of Figure 13. We estimate that the date of $R$-band maximum was 30 Sep. 2003, which means our two spectra were obtained at phases of -11 and +22 days; hence, we show the $-10 \mathrm{~d}$ and $+20 \mathrm{~d}$ average spectra. The peculiarity of SN 2003id is apparent here as well: our spectra deviate significantly from both $\mathrm{SNe} \mathrm{Ib}$ and SNe Ic spectra at several wavelengths. As above, we find that the putative He I $\lambda 6678$ line is SN Ib-like, while the lack of a He I $\lambda 7065$ line is SN Ic-like, and the extreme widths of the Ca II and $5700 \AA$ features are unlike both.

The light curves provide an additional wrinkle: SN 2003id distinctly shows a double-peaked evolution, with a rapid decline from the blue first peak followed by a rise to the second maximum a few days later. There is no evidence from the later spectrum that the second peak arises from interaction with dense circumstellar interaction (i.e., we find no narrow emission lines). Most $\mathrm{SNe} \mathrm{Ib}$ and SNe Ic do not exhibit double-peaked light curves like those of SN 2003id, though similar behavior is often observed in SNe IIb. A very small number of double-peaked SNe Ib have been discovered (e.g., SNe 2005bf and 2008D; Tominaga et al. 2005; Folatelli et al. 2006; Modjaz et al. 2009), as has one doublepeaked SN Ic (Taddia et al. 2016a). However, these events had early peaks which were notably less bright than their main peaks, they exhibited a diversity of different peak absolute magnitudes, and none show the peculiar $5700 \AA$ feature of SN 2003id.
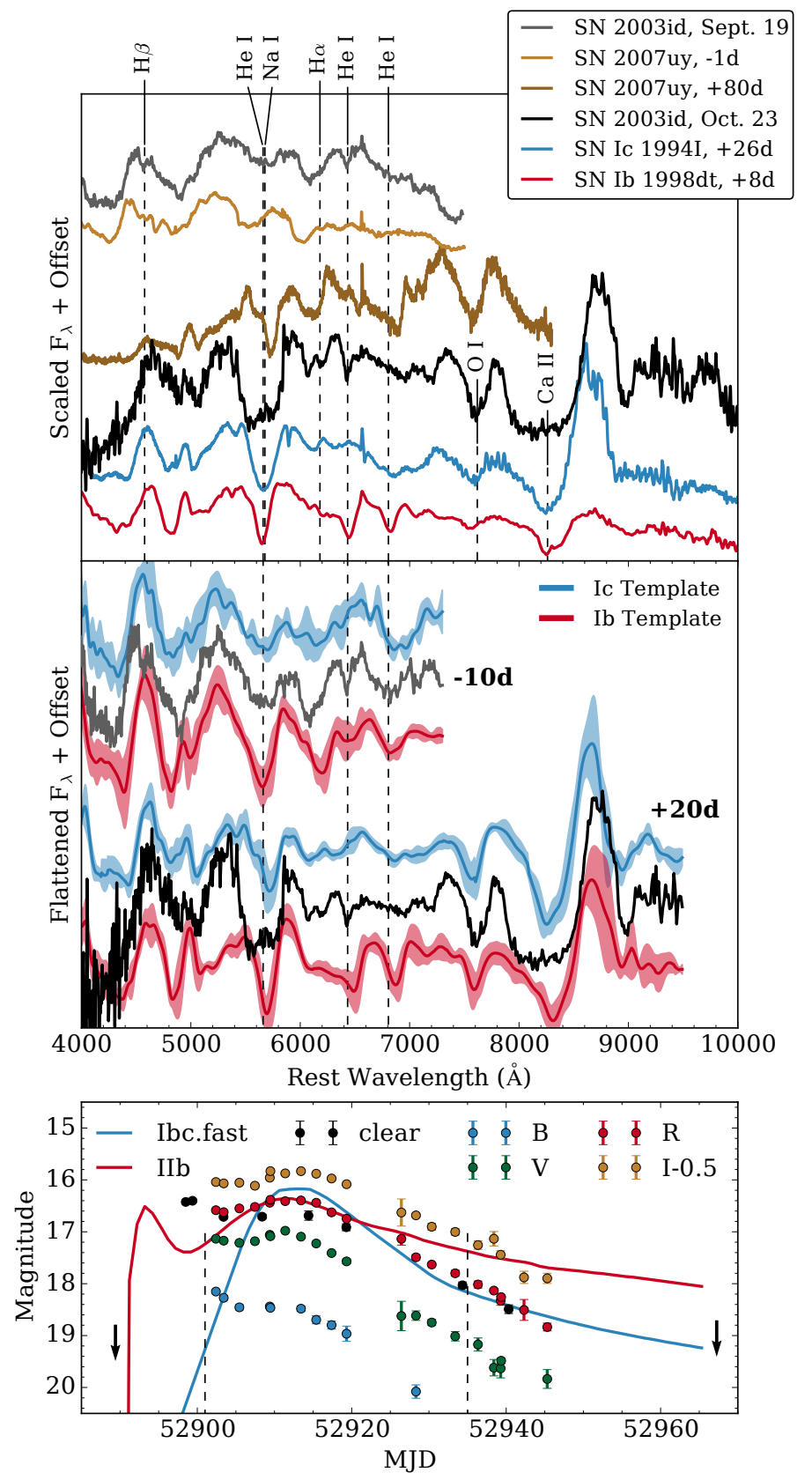

Figure 13. Top: spectra of SN 2003id smoothed with a $20 \AA$ Gaussian kernel and compared to spectra of the peculiar Type Ib SN 2007uy (Modjaz et al. 2014), the normal Type Ic SN 1994I (Filippenko et al. 1995), and the normal Type Ib SN 1998dt (Matheson et al. 2001). Putative hydrogen and helium lines are marked at $v=17,500 \mathrm{~km} \mathrm{~s}^{-1}$ and $11,000 \mathrm{~km} \mathrm{~s}^{-1}$, respectively. Middle: the continuumnormalized spectra of SN 2003id compared to the $-10 \mathrm{~d}$ and $+20 \mathrm{~d}$ average spectra of SNe Ib and SNe Ic from Liu et al. (2016), with the standard deviation shown as shaded regions and He I lines marked as above. Bottom: filtered light curves of SN 2003id, with phases of spectra marked by the dashed lines. Template SN IIb and "fast" SN Ib/c light curves from L11 are overplotted.

Given the lack of He I $\lambda 7065$ and the uncertain (and certainly peculiar) He I $\lambda 6678 \AA$ and He I $\lambda 5876 \AA$ lines, 
we classify SN 2003id as a peculiar and double-peaked SN Ic. We note that this object appears to be different from the other stripped-envelope SNe in this sample.

\subsubsection{SN 2004bm $(I b c-p e c / I I b \rightarrow I I b / I I b-p e c)$}

SN 2004bm was discovered in NGC 3437 (Armstrong et al. 2004) and originally classified as a SN Ic (though with some uncertainty; Foley et al. 2004a). L11 note that the light curve shows a dip. Though the data are sparse, the SN occurred near the core of its host galaxy, and this conclusion depends upon only one data point out of four total, our rereduction of the light curve also shows a dip indicated by the second detection - see Figure 14. Similar light-curve behavior has been observed in Type IIb SNe (e.g., L11; Arcavi et al. 2012), and L11 used the light curve to argue that SN 2004bm was a SN IIb.
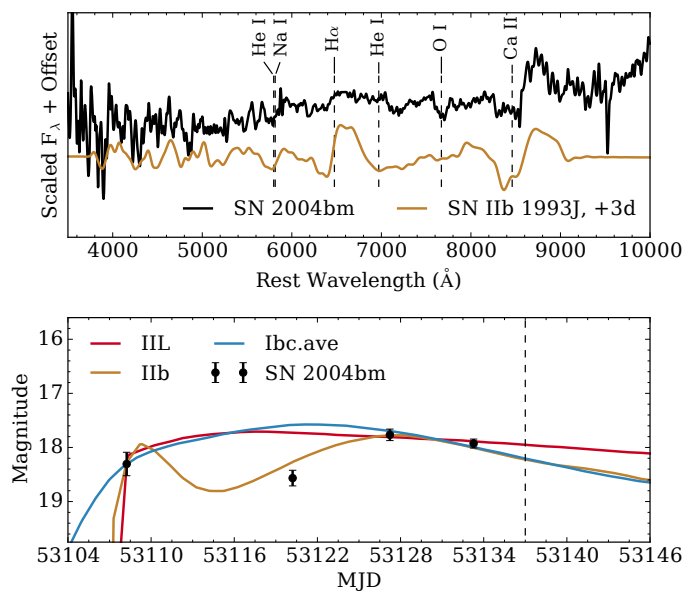

Figure 14. Top: spectrum of SN 2004bm smoothed with a Gaussian kernel $40 \AA$ wide and compared to the nearmaximum spectrum of the Type IIb SN 1993J (reddened by $E(B-V)=0.4 \mathrm{mag}$ for comparison; Matheson et al. 2000). Bottom: the light curve compared to the Type IIL, IIb, and average Ib/c template light curves from L11. A single data point indicates a light-curve dip and argues for a Type IIb classification. The date of the spectrum is marked by the vertical line.

The only spectrum we have of this $\mathrm{SN}$ is the one used for the original classification and it is of low quality - SNID does not provide a clear classification, but it does indicate that the best cross-correlations are with spectra of SNe IIb and IIP (though the phases are in disagreement). The spectrum of SN 2004bm does not correlate with spectra of Type Ic SNe well. Narrow $\mathrm{Na}$ I D absorption at the host-galaxy redshift is apparent but unresolved and very noisy, indicating moderate host-galaxy reddening atop the MW contribution of $E(B-V)=0.0159 \mathrm{mag}$. There are few spectral features in our spectrum, though we identify Ca II P-Cygni profiles, faint $\mathrm{O}$ I absorption, and a very shallow $\mathrm{H} \alpha \mathrm{P}$ -
Cygni line - see Figure 14.

Based on the above discussion, we prefer a classification of Type IIb for SN 2004bm, but the $\mathrm{H} \alpha$ line in the spectrum of SN $2004 \mathrm{bm}$ is much too weak for a normal Type IIb SN. We also note that, if we discard the second photometric data point, the light curve of SN 2004bm is well-fit by normal Type II events or by stripped-envelope events.

\subsection{7. $S N 2004 c c(I c \rightarrow I b / I c)$}

SN 2004cc was discovered in NGC 4568 (Monard \& Li 2004). Matheson et al. (2004d) note the strong reddening toward SN2004cc and classify it as a Type I SN, though they prefer no subtype, while Foley et al. (2004b) present a SN Ic classification.

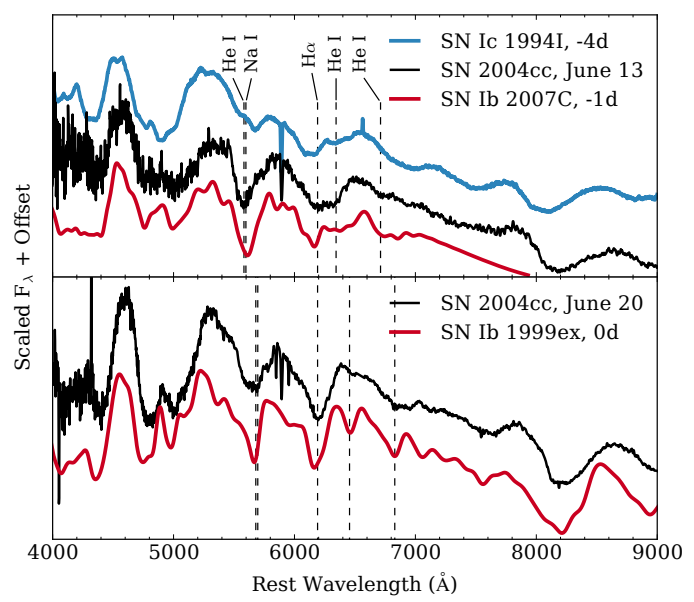

Figure 15. Top: spectrum of SN 2004cc observed on UT 2004-06-13 alongside premaximum spectra of SN Ic 1994I and SN Ib 2007C (Filippenko et al. 1995; Modjaz et al. 2014), and we mark He I lines at a velocity of $15,000 \mathrm{~km} \mathrm{~s}^{-1}$. Bottom: spectrum of SN 2004cc observed on UT 2004-06-20 and a spectrum of the Type Ib SN 1999ex (Hamuy et al. 2002), with the helium line marked at $10,000 \mathrm{~km} \mathrm{~s}^{-1}$, a deceleration consistent with the normal evolution of helium-line velocities in SNe Ib (Liu et al. 2016). Both spectra of SN 2004cc have been dereddened by $E(B-V)=1.0$ mag.

There is little MW reddening toward SN 2004cc $(E(B-V)=0.0279 \mathrm{mag})$, but the (unresolved) Na I D lines in our spectra indicate strong host-galaxy dust obscuration. The EW measured from these lines is well outside the relations of Poznanski et al. (2012), and so we only roughly estimate the total reddening, adopting $E(B-V)=1.0 \mathrm{mag}$ for visual comparisons.

SNID identifies reasonable correlations between the spectra of SN 2004cc and the spectra of both SNe Ib and SNe Ic, strongly disfavoring all other types and slightly preferring the Ib label over Ic. Unfortunately, we have only a single photometric detection of the SN, so there is little independent information about the phases of these spectra. 
Figure 15 shows that the later spectrum of SN 2004cc matches that of SN Ib 1999ex quite well, while the earlier spectrum matches that of SN Ic 1994I. Weak $\mathrm{H} \alpha$ detections have been claimed for both of these events (Branch et al. 2006). Though hydrogen absorption may be present, we trust the SNID result (which prefers a Type Ib or Ic label, rather than a IIb), and we do not consider a Type IIb label for SN 2004cc - see the discussion in $\S 3$. Note also that, if the identification of the $\mathrm{H} \alpha$ line in Figure 15 is correct, it exhibits a much faster (and unchanging) Doppler velocity $\left(\sim 17,000 \mathrm{~km} \mathrm{~s}^{-1}\right)$ than the He I lines (which are at $\sim 15,000$ and $\sim 10,000 \mathrm{~km} \mathrm{~s}^{-1}$ on June 13 and June 20, respectively). This behavior is peculiar but not unique for this feature in strippedenvelope SNe (Liu et al. 2016).

The spectra of SN 2004cc present another puzzle. The early-time spectrum appears to show a strong He I $\lambda 6678$ line (and a strong He I/Na I blend near $5500 \AA$ ), but very little He I $\lambda 7065$ absorption. It is difficult to physically explain a strong He I $\lambda 6678$ absorption line without a similarly strong $\lambda 7065$ line; other ions may be contributing to this feature. Just one week later the He I $\lambda 6678$ line has disappeared, though the width of the line near $5500 \AA$ implies that He I $\lambda 5876$ is still present. Given the above uncertainties and the weakness of the He I $\lambda 7065$ line, we assign SN 2004cc the Ib/Ic label. Interestingly, Wellons et al. (2012) present a variable and long-lasting radio light curve, indicating the presence of a complex circumstellar medium near this object.

\subsection{8. $S N$ 2005H $(I I \rightarrow I I / I I b)$}

SN 2005H was discovered in NGC 838 (Graham et al. 2005) and classified as a Type II SN based upon a noisy spectrum (Pastorello et al. 2005a). Very few data exist on SN 2005H, and most of the spectra are entirely dominated by host-galaxy light. However, we were able to obtain the original classification spectrum (Harutyunyan et al. 2008) and we find clear detections of $\mathrm{H} \alpha$ absorption and $\mathrm{P}-\mathrm{Cygni}$ profiles of $\mathrm{H} \beta$ and $\mathrm{Na}$ I on an otherwise smooth blue continuum; see Figure 16.

SNID shows this spectrum to be more similar to spectra of young SNe II than SNe IIb. We have very little information on the light-curve evolution of this object - the SN was discovered very near the bright ( $R \approx 12-14 \mathrm{mag}$ ) core of the host and our data are badly contaminated by galaxy light. Other than the discovery image we have no clear detections, only uninformative upper limits.

\subsection{9. $S N$ 2005mg $(I I \rightarrow I I / I I b)$}

SN 2005mg was discovered in UGC 155 (Newton \& Puckett 2005) and classified as a heavily reddened young Type II SN, with detections of $\mathrm{H} \alpha$ and $\mathrm{H} \beta$ in the noisy spectrum (Modjaz et al. 2005). We have a spectrum ob-

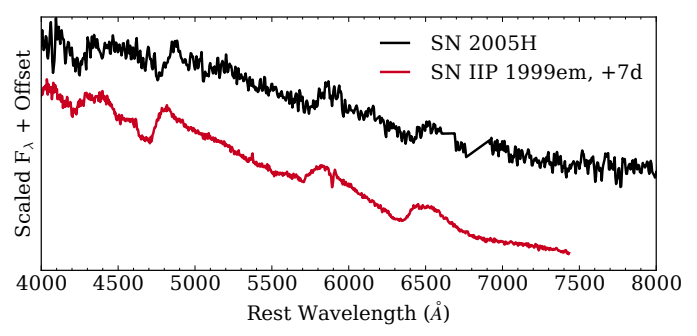

Figure 16. Spectrum of SN $2005 \mathrm{H}$ alongside a spectrum of the Type IIP SN 1999em (Leonard et al. 2002b).

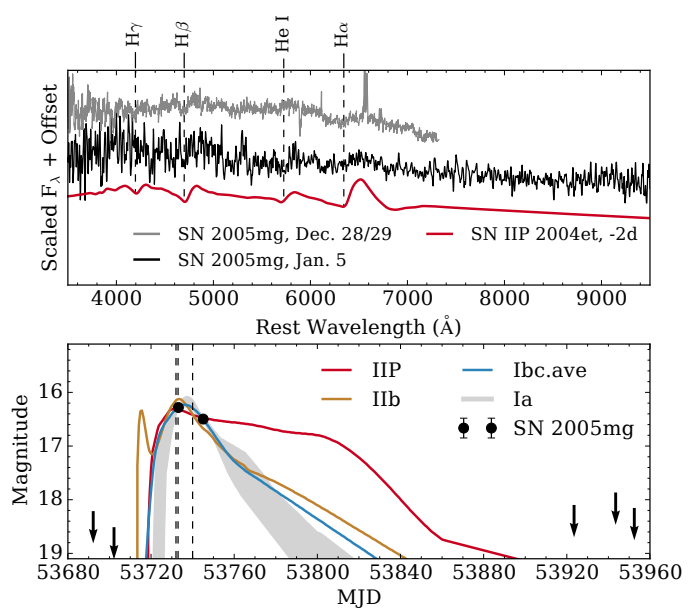

Figure 17. Top: spectra of SN $2005 \mathrm{mg}$ alongside a spectrum of the Type IIP SN 2004et (Sahu et al. 2006). In blue we show the co-addition of the traces obtained from plots of the two spectra from Dec. 2005, while in black we show the full spectrum from Jan. 2006. Bottom: the light curve compared to template light curves from L11, with the dates of spectra shown with dashed lines.

tained one week later, but unfortunately we were unable to locate a digital copy of the spectrum cited above. We did, however, locate plots of both the classification spectrum and another one obtained the night prior, both in image form. ${ }^{14}$ Using an online tool, ${ }^{15}$ we traced the spectra from these plots and consider the results in our analysis.

All three spectra exhibit extremely low S/N and SNID identifies no good matches. The KAIT light curve is similarly uninformative with only two detections and is consistent with template light curves of all types; see Figure 17. However, the spectra do appear to corroborate the Modjaz et al. (2005) classification of SN 2005mg as a Type II SN, with plausible detections of weak $\mathrm{H} \alpha$ and $\mathrm{H} \beta$ lines showing broad P-Cygni profiles. We therefore consider the original classification robust though we

\footnotetext{
14 https://www.cfa.harvard.edu/supernova/spectra/

15 http://arohatgi.info/WebPlotDigitizer/app/
} 
cannot determine whether SN 2005mg was a Type IIb or a normal Type II SN, and so we label it as II/IIb (unsure).

\subsubsection{SN 2006eg $(I c \rightarrow I I b / I b / I c / I c-B L)$}

SN 2006eg was discovered in an anonymous galaxy (Madison \& Li 2006) and classified as a SN Ib/c (Foley et al. 2006). SNID, GELATO, and Superfit identify no good matches, but the best cross-correlations are with spectra of SNe Ic-norm and SNe Ic-BL. The MW reddening toward SN 2006eg is small $(E(B-V)=0.0933 \mathrm{mag})$, and though the spectrum of SN 2006eg is noisy it does not appear that there could be more than a modest degree of host-galaxy reddening — we detect no narrow Na I D lines at the host galaxy's redshift. Our sparse and noisy light curve is most similar to that of a Type IIL SN, but it is also consistent with a stripped-envelope classification.
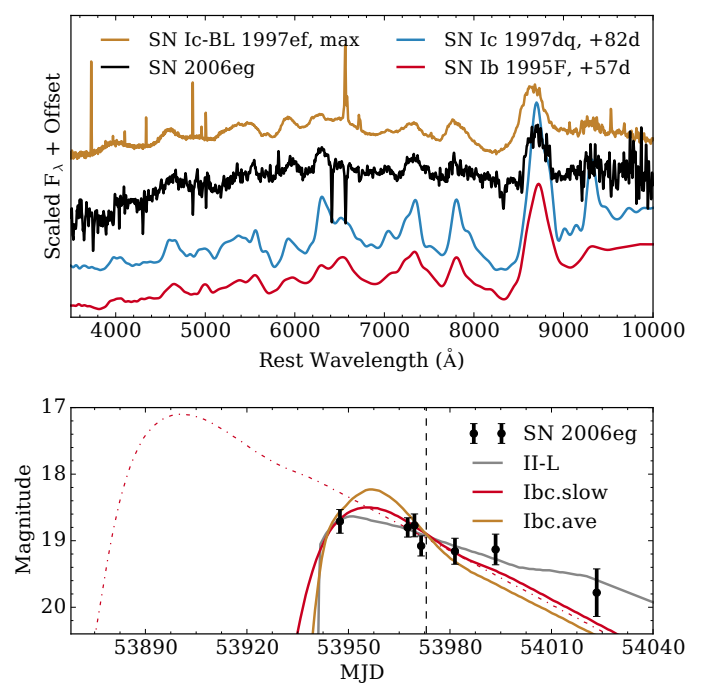

Figure 18. Top: spectrum of SN 2006eg (smoothed with a $20 \AA$ Gaussian kernel) compared to that of the SN Ic-BL $1997 \mathrm{ef}$, SN Ic 1997dq, and SN Ib 1995F (Matheson et al. 2001; Modjaz et al. 2014). Bottom: the light curve, with the date the spectrum was taken marked, alongside template light curves from L11. Our last prediscovery upper limit was $173 \mathrm{~d}$ before the first detection; though we show comparison light curves assuming the peak was observed, the true peak could have occurred months before discovery. The red dashdot line shows the Ibc.slow template of L11 offset by 55 days, illustrating how the peak may have occurred prediscovery.

SN 2006eg had a peak measured magnitude of $-14.86 \pm 0.23 \mathrm{mag}$ (unfiltered; L11), which makes the event an underluminous outlier from both the SN IcBL and normal SN Ib/Ic populations (L11, Drout et al. 2011). However, as Figure 18 shows, the true peak could have occurred before discovery and the event may have been significantly more luminous at the true (unobserved) peak.
Our spectrum of SN 2006eg is not only noisy, it also exhibits a low contrast between the continuum and the SN features - this event occurred near the center of its spiral host galaxy, and it appears that our spectrum suffers from significant host-galaxy contamination (the strong, narrow absorption features are likely badly subtracted host-galaxy emission lines). It has been our experience that SNID sometimes mistakenly prefers a SN Ic-BL classification for low-contrast spectra, and so we are hesitant to assign much belief to that result.

The He I lines that mark the difference between SNe Ib and SNe Ic are, in general, time-dependent: they are most apparent soon after peak and generally fade completely away by $\sim$ 50-70 days (Modjaz et al. 2014; Liu et al. 2016). These types can be difficult to differentiate at these ages, and $\mathrm{SNe} \mathrm{Ic}, \mathrm{Ib}$, and IIb all show strong features at the same wavelengths as those in SN 2006eg's spectrum. It is likely that our spectrum of SN 2006eg shows a nearly nebular SN IIb, Ib, or Ic "watered down" by host-galaxy contamination. If SN 2006eg was a mostly normal stripped-envelope SN discovered late, then its light curve was slowly declining, but it was not an extreme outlier from the diverse late-time decline rates observed for these SNe (e.g., Lyman et al. 2016).

Without better data, it is very difficult to firmly label SN 2006eg - our spectrum shows it was not a Type Ia or Type II SN, but it could have been a SN IIb, Ib, Ic, or Ic-BL.

\subsection{SNe $I a$}

We follow Silverman et al. (2012) when identifying the subtypes of SNe Ia, and in most cases simply duplicate the classifications from their Table 7 . We propose some updated subtype classifications from L11, but no objects appear to be misclassified as SNe Ia, nor does it appear that any events labeled otherwise should be reclassified as SNe Ia.

We update the classifications of SNe 1999bh and 2002es, labeling them with the subtype "Ia-02es" as identified by Ganeshalingam et al. (2012). These events are subluminous and exhibit low expansion velocities, sharing properties with both the SN Ia-2002cx (i.e., SN Iax; Foley et al. 2013) and SN Ia-1991bg subtypes. Though L11 note that these two events may form their own subtype, they include them with the SNe Iax as the properties of the subtype were only partially understood at the time (subsequent work has furthered our understanding; e.g., Cao et al. 2015; White et al. 2015; Cao et al. 2016). Foley et al. (2013) show that SNe Iax display a wide range of peak luminosities, from the extremely subluminous SN 2008ha $\left(M_{V} \approx-14.2 \mathrm{mag}\right)$ up into the range of typical SNe Ia $\left(M_{V} \approx-18.5 \mathrm{mag}\right)$, and the rate calculations of L11 did not account for the 
low-luminosity members of this class and therefore underestimated the true rate of these events.

We also update several events previously labeled SNe Ia 1991T to the "SN Ia-1999aa" subtype (Garavini et al. 2004), a subclass that falls in between SNe Ia-norm and SNe Ia-1991T and another distinction intentionally not included in L11 (see Li et al. 2001). Three of these events (SNe 1998es, 1999aa, and 1999dq) were previously given a Ia-99aa label by Silverman et al. (2012). The spectral evolution of SN 1999ac was studied in detail by Garavini et al. (2005), who note that early-time spectra are similar to those of SN 1999aa with relatively weak silicon absorption, but SNID identifies both premaximum and postmaximum spectra as SN Ia-norm (Silverman et al. 2012, though Ia-99aa templates also provide reasonable fits) and subtle peculiarities exist throughout this object's evolution. Owing to this peculiarity, we give this event equal weights in the Ia-norm and Ia-99aa subclasses.

SN $2001 \mathrm{~V}$ is grouped among the "shallow silicon" events by Branch et al. (2009), and the premaximum spectra of SN 2001V published by Blondin et al. (2012) are strongly classified as 99aa-like by SNID. The data on SN $2006 \mathrm{~cm}$ are somewhat less conclusive and the early evolution is not well constrained. The spectra show strong $\mathrm{Na}$ ID absorption features from the host UGC 11723 and they are noticeably reddened by host-galaxy dust (Blondin et al. 2006; Sternberg et al. 2011). SNID prefers a SN Ia-normal classification for SN 2006cm (Silverman et al. 2012), but the spectra also exhibit good matches to those of Ia-99aa objects and the silicon absorption features are weaker than those in the SNIDpreferred SN Ia-norm templates. We give SN $2006 \mathrm{~cm}$ equal weights in the SN Ia-norm and SN Ia-99aa subclasses.

Finally, SN 2004bv is classified as a SN Ia-91T event by SNID, but unfortunately the only existing premaximum spectrum of this SN does not extend to sufficiently blue wavelengths to capture the Ca II H\&K lines, which are the strongest indicator of a SN 1991T-like event at young epochs (Silverman et al. 2012, see their Fig. 5), and so this classification is somewhat suspect and this event may also have been SN 1999aa-like. These updates indicate that $91 \mathrm{~T} / 99$ aa-like events exhibit a continuum of spectroscopic properties, with normal SNe Ia at one end and SN 1991T-like events at the other extreme; most "shallow silicon" events fall somewhere in between.

\subsection{SNe Ic-BL}

L11 grouped the broad-lined SNe Ic (SN Ic-BL, sometimes associated with gamma-ray bursts; e.g., Woosley \& Bloom 2006) into the SN Ic-pec subclass, though they noted in the text that SN 2002ap is a member of that group (e.g., Mazzali et al. 2002). As discussed in §4.2,
SNe 2002jj and 2006eg may plausibly also be of Type Ic-BL.

\subsection{SN 1987A-like SNe}

As discussed in $\$ 4.1 .6$, SN 2005io was very likely a SN 1987A-like event (for reviews of SN 1987A and related events, see, e.g., Arnett et al. 1989; McCray 1993). The LOSS volume-limited sample also includes the SN 1987A-like SNe 2000cb and 2005ci (Kleiser et al. 2011). All of these objects were grouped with the Type IIP SNe by L11.

\subsection{Ca-Rich Transients}

There are three examples of the recently identified class of "Ca-rich" SNe in our sample: SNe 2003H, 2003dr, and 2005E (e.g., Filippenko et al. 2003; Perets et al. 2010; Kasliwal et al. 2012; Foley 2015). All three were identified by Perets et al. (2010). Though discussed within the text as Ca-rich events, these three SNe were labeled SN Ibc-pec by L11 and were grouped with the other stripped-envelope SNe in their analysis.

Though the provenance of these events is not fully understood, it now seems likely that they do not arise from the core collapse of massive stars. Removing these three events from the sample of core-collapse SNe slightly reduces the ratio of stripped-envelope SNe relative to Type II SNe. Figure 19 shows spectra of all three Ca-rich SNe in the sample. We note that the photospheric spectra of these events are extremely similar to those of normal $\mathrm{SNe} \mathrm{Ib}$; it is their nebular spectra, their rapid evolution, and their low peak luminosities that primarily differentiate these events.

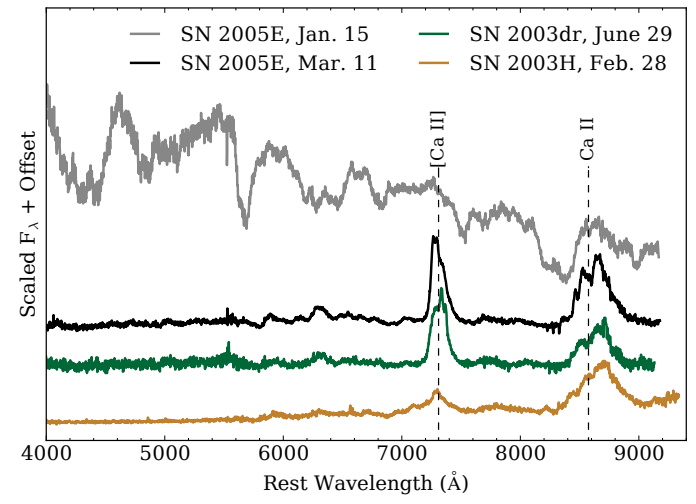

Figure 19. Spectra of the Ca-rich objects in our sample. For SN 2005E we present photospheric and nebular spectra; for the other objects we only have nebular spectra.

\subsection{Type IIn SNe and SN Impostors}

Type IIn SNe are hydrogen-rich SNe that exhibit narrow lines in their spectrum - indicative of dense circumstellar material surrounding the progenitor at the time 
of explosion (see, e.g., Filippenko 1989; Schlegel 1990; Chugai 1991; Smith 2014). There were seven Type IIn SNe in the sample identified by L11, but two of those (SNe 2002bu and 2006bv) were reclassified as SN impostors (luminous but nonterminal outbursts from massive stars) by Smith et al. (2011b). We group SNe 2002bu and 2006bv with the five other SN impostors from the original sample and do not include them when calculating the relative fractions of SNe.

\subsection{SNe That Lack Spectra}

Every object in the L11 volume-limited sample was originally classified spectroscopically and announced through CBETs, but we have not been able to track down spectra for three events: see Table 2 and Figure 20. One of these (SN 2002ds) exhibits a light curve with a pronounced plateau, which corroborates the original CBET classification of a Type II SN. The light curve of SN 2003bw, however, does not rule out the possibility that this event was a low-hydrogen Type IIb SN (assuming that the hydrogen detection announced in the CBET is robust; Hamuy 2003). For SN 2006bv we adopt the SN impostor reclassification of Smith et al. (2011b).

Table 2. SNe That Lack Spectra

\begin{tabular}{c|ccc}
\hline \hline Name & Previous (L11) & This Work & Ref. \\
\hline SN 2002ds & IIP & II & 1 \\
SN 2003bw & IIP & II/IIb & 2 \\
SN 2006bv & IIn & impostor & 3,4 \\
\hline
\end{tabular}

References: [1] Li et al. (2002); [2] Hamuy (2003); [3] Sehgal et al. (2006); [4] Smith et al. (2011b)

\section{UPDATED FRACTION CALCULATIONS}

Figure 21 and Table 3 summarize our updated fraction calculations. We follow L11 to estimate our uncertainties, running $10^{6}$ Monte Carlo realizations of the sample assuming Poisson statistics and the control-time corrections from L11, Leaman et al. (2011), and Li et al. (2011a). (Note that the stated uncertainties are statistical only.) We do not recalculate the control times for each event after these updated classifications. Almost all of our updates are swaps between stripped-envelope subtypes which show very similar light curves, and so the control-time calculations should change very little. For events with more than one possible classification listed in Table 1 we assign a fractional weight to each given the relative frequencies of the subtypes among the wellclassified events.

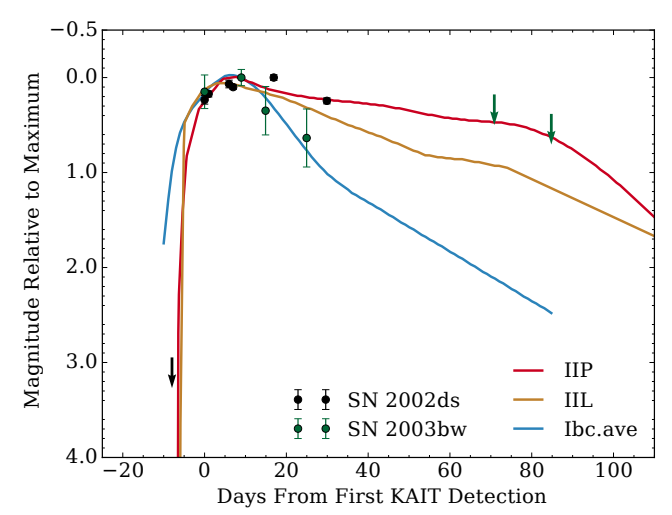

Figure 20. Unfiltered light curves for two $\mathrm{SNe}$ for which we have not been able to collect spectra, along with template light curves from L11 for comparison. Upper limits are shown with arrows. For SN 2002ds, the CBET classification of a Type II SN with a hydrogen-recombination plateau is robustly supported. The light curve of SN 2003bw appears to match the stripped-envelope template from L11 better than the Type II templates, but the data are noisy and are consistent with either classification. SN 2006bv (not shown) was likely a SN impostor; see Smith et al. (2011b).

Many authors, based on both theory and observation, argue for trends in the relative SN rates as a function of metallicity (e.g., Modjaz et al. 2008; Arcavi et al. 2010; Modjaz et al. 2011; Kelly \& Kirshner 2012; Yoon 2015). Adopting the metallicity-luminosity relation of Garnett (2002) and noting that the galaxies hosting core-collapse SNe within the LOSS sample cover a range of luminosities of $M_{K} \approx-22$ to $-25 \mathrm{mag}$, Smith et al. (2011a) estimate metal abundances of $\sim 0.5-2.0 \mathrm{Z}_{\odot}$ for these galaxies, and our results are therefore applicable to roughly that range. Graur et al. (2016a,b) use the LOSS sample and our updated classifications to examine correlations between SN rates and host galaxy properties in more detail, including the stellar masses, specific star-formation rates, and oxygen abundances (i.e., metallicities) of the host galaxies. Several authors have shown that local measures of host-galaxy metallicities are more informative than global ones (e.g., Modjaz et al. 2008; Anderson et al. 2010; Modjaz et al. 2011; Anderson et al. 2016); a study of the explosion-site metallicities of the LOSS sample is a worthy endeavor we leave to future work.

As with L11, our calculations for several of the rarer subtypes suffer from the effects of small-number statistics, but Table 3 and Figure 21 indicate an important update: the percentage of SNe Ic is reduced while the percentage of $\mathrm{SNe} \mathrm{Ib}$ is increased. Adopting our updated classifications, $83 \%$ of our Monte Carlo trials indicate that normal SNe Ib are more common than normal SNe Ic, while $99 \%$ of the trials using the L11 classifications indicate the opposite.

L11 and Smith et al. (2011a) found that SNe Ic are more than twice as common as $\mathrm{SNe} \mathrm{Ib}$ (grouping the $\mathrm{SNe}$ 

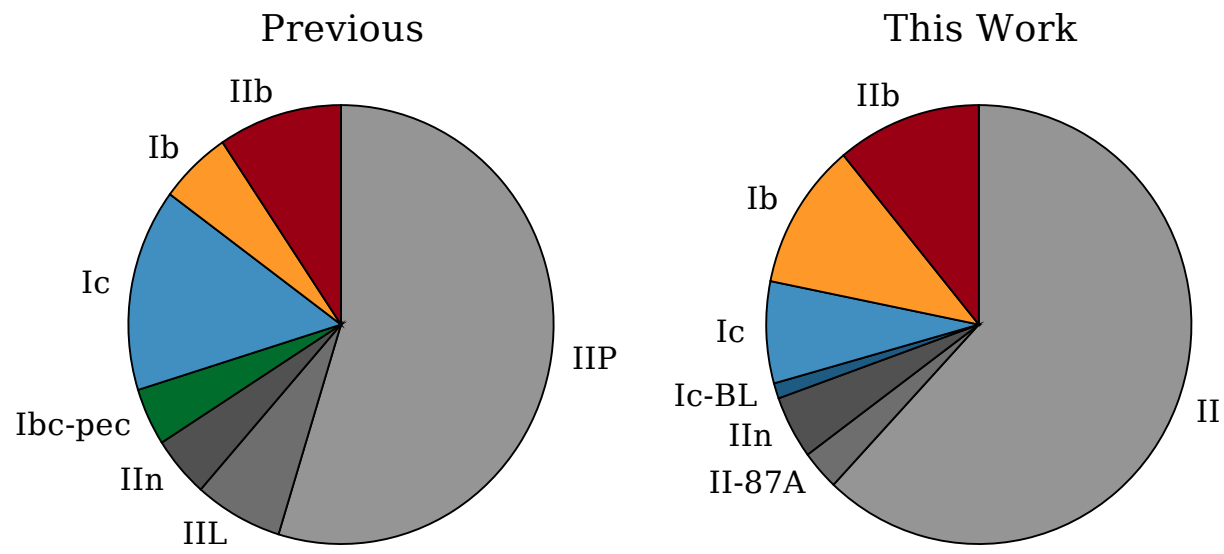

Figure 21. Relative fractions of core-collapse SN types within a volume-limited sample using the original classifications from L11 (left) compared to the updated classifications presented here (right). Subtypes are color-coded along with the other members of their major type, and the "peculiar" subtype labels are grouped with the appropriate "normal" events (except for the SN Ibc-pec group of L11, which included both SNe Ic-BL and Ca-Rich transients). All fractions are listed in Table 3 and any objects listed in Table 1 with more than one possible classification are given a fractional weight in each class, as described in $\S 5$.

Ic-BL with the SNe Ic, which only affects these rates by a small amount). L11 calculate a ratio of SNe Ic/SNe Ib $=54.2 \pm 9.8 \% / 21.2_{-7.7}^{+8.4} \%=2.6 \pm 1.1$, while Smith et al. (2011a, excluding SNe from highly inclined galaxies) calculated $\mathrm{SNe} \mathrm{Ic} / \mathrm{Ib}=14.9_{-3.8}^{+4.2} \% / 7.1_{-2.6}^{+3.1} \%=2.1 \pm 1.1$ (in all cases the errors listed are statistical only, and were derived from Monte Carlo simulations similar to those described above).

We now calculate a ratio of normal SNe Ic to normal $\mathrm{SNe} \mathrm{Ib}$ of $0.6 \pm 0.3$ and, if we include the SNe Ic-BL and other peculiar subtypes with the normal $\mathrm{SNe} \mathrm{Ib}$ and Ic, we find a (SN Ic+Ic-BL+Ic-pec)/SN Ib ratio of $0.8 \pm 0.4$.

This update to the population fractions is driven by our reclassifications of seven stripped-envelope events. First, we relabeled four events from a Ic subtype to a Ib or IIb subtype (SNe 2001M, 2001ci, 2004C, and 2005lr). In each of these cases, the need for reclassification is easily understood: three of these events had spectra severely reddened by host-galaxy dust and were originally classified by eye without the aid of SNID, and one showed only weak He I lines in the spectrum (SN $2001 \mathrm{M}$ ). Second, we created the SN Ib/Ic (unsure) category, which includes an additional two events that show weak He I lines with some uncertainty on their identification (SNe 2002jz and 2004cc) and one event with only sparse and noisy observations (SN 2006eg). If we assume that all of the SNe in the latter category deserve the Ic label, our Monte Carlo trials indicate that normal $\mathrm{SNe} \mathrm{Ib}$ and SNe Ic (excluding peculiar subtypes) occur at similar rates: $\mathrm{SNe} \mathrm{Ic} / \mathrm{Ib}=0.9 \pm 0.5$. If we rather assume that they are all $\mathrm{SNe} \mathrm{Ib}$, we get a ratio of normal $\mathrm{SNe} \mathrm{Ic} / \mathrm{Ib}=0.5 \pm 0.3$.
These results have implications for our understanding of the progenitors of stripped-envelope $\mathrm{SNe}$, as we discuss below, and may affect other works that use the LOSS rates as input (e.g., Foley \& Mandel 2013).

\section{PROGENITOR CONSTRAINTS ON STRIPPED-ENVELOPE SNE}

Wolf-Rayet (WR) stars have long been discussed as Galactic analogues of SN Ib/c progenitors (e.g., Meynet \& Maeder 2003; Crowther 2007), though many authors have argued that binary stars which undergo mass loss via Roche-lobe overflow before core collapse are likely the most common SN Ib/c progenitor (e.g., Podsiadlowski et al. 1992; Smartt 2009; Smith et al. 2011a; Eldridge et al. 2013). Regardless, stellar modeling efforts have found it difficult to match the SN Ic/Ib fractions presented by L11 and Smith et al. (2011a), which demand more SN Ic progenitors (stars that lose both their hydrogen envelope and a large fraction of their helium envelopes) than $\mathrm{Ib}$ progenitors (stars that lose just the hydrogen; e.g., Georgy et al. 2009; Yoon et al. 2010; Yoon 2015), though some success has been achieved by invoking rapid rotation of the progenitors (e.g., Cao et al. 2013; Groh et al. 2013b).

To address this putative issue, some authors have proposed that some amount of helium in SNe Ic may be "hidden" and remain neutral if the ${ }^{56} \mathrm{Ni}$ (which provides nonthermal excitations via radioactive decay) is insufficiently mixed with the helium-rich ejecta (e.g., Dessart et al. 2011, 2012). Comparisons to observation do not find evidence for large amounts of hidden helium in $\mathrm{SNe}$ Ic, however, and it is unclear from the models how much helium could truly be hidden in this way (e.g., Hachinger 
Table 3. Updated Relative SN Fractions in a Volume-Limited Survey

\begin{tabular}{|c|c|c|c|}
\hline Type & Previous & This Work & Difference \\
\hline & \multicolumn{2}{|c|}{ Core Collapse } & \\
\hline II & $68.9_{-6.0}^{+6.0}$ & $69.6_{-6.7}^{+6.7}$ & - \\
\hline \multirow[t]{2}{*}{$\mathrm{IIb}+\mathrm{Ib}+\mathrm{Ic}$} & $31.1_{-4.6}^{+4.6}$ & $30.4_{-4.9}^{+5.0}$ & - \\
\hline & \multicolumn{2}{|c|}{ Stripped Envelope } & \\
\hline $\mathrm{IIb}$ & $27.6_{-9.1}^{+9.1}$ & $34.0_{-11.1}^{+11.1}$ & +6.3 \\
\hline IIb-pec & - & $2.0_{-2.0}^{+1.5}$ & - \\
\hline $\mathrm{Ib}$ & $16.1_{-6.6}^{+6.8}$ & $35.6_{-11.4}^{+11.4}$ & +19.5 \\
\hline Ib-pec & - & - & - \\
\hline Ibc-pec ${ }^{\alpha}$ & $12.4_{-5.6}^{+5.9}$ & - & - \\
\hline Ic & $41.1_{-11.4}^{+11.5}$ & $21.5_{-8.6}^{+8.6}$ & -19.6 \\
\hline Ic-pec & $2.8_{-2.8}^{+2.6}$ & $3.2_{-3.2}^{+3.1}$ & - \\
\hline \multirow[t]{2}{*}{ Ic-BL } & - & $3.7_{-3.7}^{+2.9}$ & - \\
\hline & \multicolumn{2}{|c|}{ Hydrogen Rich } & \\
\hline $\mathrm{II}^{\beta}$ & $93.2_{-11.3}^{+11.5}$ & $89.1_{-10.9}^{+10.9}$ & - \\
\hline II-87A & - & $4.2_{-2.7}^{+2.4}$ & - \\
\hline IIn & $6.8_{-2.9}^{+3.0}$ & $6.7_{-2.9}^{+3.0}$ & - \\
\hline
\end{tabular}

Relative fractions of core-collapse $\mathrm{SNe}$ in the LOSS volume-limited sample, within several different subsets, expressed in percentages. In the left column we present the fractions assuming the original classifications used by $\mathrm{L} 11$, in the center column we present our updated fractions, and in the right column we highlight the most notable updates.

${ }^{\alpha}$ L11 included SNe Ic-BL and Ca-Rich transients with the Ibc-pec class. In our updated fractions we list the SNe Ic-BL seperately, and we do not group the Ca-Rich events with core-collapse SNe.

${ }^{\beta}$ Including the II-L and II-P subclasses of L11.

et al. 2012; Taddia et al. 2015; Liu et al. 2016). Our updated stripped-envelope fractions argue that this problem is less egregious than previously indicated.

Other discrepancies have arisen within the single WRlike progenitor scenario. The observed ejecta masses of normal SNe Ib/c $\left(M_{\mathrm{ej}} \approx 2.0-4 \mathrm{M}_{\odot}\right.$; Drout et al. 2011; Cano 2013; Lyman et al. 2016) are not in good agreement with the estimated masses of WR stars at the time of core collapse $\left(M \gtrsim 10 \mathrm{M}_{\odot}\right.$; Meynet \& Maeder 2003; Yoon 2015), assuming that most $\mathrm{SNe} \mathrm{Ib} / \mathrm{c}$ produce neutron star remnants rather than black hole remnants. Note that SNe Ic-BL may have larger ejecta masses, and so this reasoning holds for normal $\mathrm{SNe} \mathrm{Ib} / \mathrm{c}$ only (Cano 2013). In addition, the rates of $\mathrm{SNe} \mathrm{Ib} / \mathrm{c}$ compared to those of Type II SNe are inconsistent with WR star progenitors (the incidence rate of WR stars is too low to explain the high fraction of $\mathrm{SNe} \mathrm{Ib} / \mathrm{c}$; e.g., Smith et al. 2011a), and the search for $\mathrm{SN} \mathrm{Ib/c} \mathrm{progenitors} \mathrm{in}$ pre-explosion images has, in several instances, ruled out normal WR stars (Eldridge et al. 2013, though see also
Groh et al. 2013b).

The binary progenitor system scenario for normal $\mathrm{SNe}$ $\mathrm{Ib} / \mathrm{Ic}$ does not suffer from the same problems. The modeled masses at the time of core collapse for post-masstransfer binary members are generally in agreement with the observed $\mathrm{SN} \mathrm{Ib/c} \mathrm{ejecta} \mathrm{masses} \mathrm{(Eldridge} \mathrm{et} \mathrm{al.}$ 2013; Yoon 2015), constraints from SN Ib/c progenitor searches are largely compatible with binary progenitors (Eldridge et al. 2013), and the identified progenitors of some SNe IIb have been shown to be the products of binary evolution (SNe 1993J and 2011dh; e.g., Maund et al. 2004, 2011; Van Dyk et al. 2011; Bersten et al. 2012).

Sana et al. (2012) show that more than $70 \%$ of O-type stars (zero-age main sequence $M \gtrsim 15 \mathrm{M}_{\odot}$ ) are formed in binary pairs that will undergo significant binary interaction (either mass gain or mass stripping) before core collapse, and so the population of core-collapse SN progenitors must necessarily be dominated by post-binaryinteraction stars. We calculate a stripped-envelope fraction of $30 \pm 5 \%$ amongst all core-collapse SNe, similar to fractions found by previous authors. As Smith et al. (2011a) note, this value is in remarkably good agreement with the $\sim 33 \%$ of O-type stars in our Galaxy found to experience envelope stripping via binary interaction before their deaths (Sana et al. 2012).

\section{CONCLUSION}

We have re-examined every SN classification within the LOSS volume-limited sample published by L11, have discussed the peculiar and rare events within the sample, and have found that several of the stripped-envelope $\mathrm{SNe}$ originally labeled as SNe Ic show clear signatures of helium and (in two cases) hydrogen. After relabeling these SNe as Type Ib or IIb appropriately, and discussing the intrinsically peculiar events and those for which we cannot assign a clear classification, we recalculate the implied fractions of these subtypes. We find that the relative fractions of Type Ia SNe, Type II SNe, and stripped-envelope SNe are unchanged, but the relative fractions between different stripped-envelope SN subtypes are.

Based on the prior spectral identifications, L11 and Smith et al. (2011a) found that SNe Ic are roughly twice as common as $\mathrm{SNe} \mathrm{Ib}$. We show that this measurement was hampered by the above misclassifications and, additionally, that the $\mathrm{SN} \mathrm{Ib} / \mathrm{SN}$ Ic ratio is strongly dependent on exactly where one draws the line between these subclasses. We find that $\mathrm{SNe} \mathrm{Ib}$ are at least as common as SNe Ic in the local universe and in fact are likely to be more common. We present a best-estimate normal $\mathrm{SN}$ Ic/SN Ib ratio of $0.6 \pm 0.3$ - i.e., spectroscopically normal SNe Ib occur in the local universe $1.7 \pm 0.9$ times more often than do normal SNe Ic. 
Other efforts (e.g., Smartt et al. 2009; Eldridge et al. 2013) found SN Ic/SN Ib ratios similar to those of L11, and we believe they may also have been plagued by systematically mislabeled stripped-envelope events. The updated stripped-envelope SN fractions published here should prove important for constraining the elusive progenitors of the various subtypes of stripped-envelope $\mathrm{SNe}$, and we hope the public release of these data will be useful when exploring this valuable sample going forward.

We thank the many observers who assisted in obtaining the data published here, especially the UC Berkeley undergraduates who have worked to discover new SNe within the KAIT data. We are grateful to J. Mauerhan, M. Graham, P. Kelly, P. Challis, R. McCray, and I. Kleiser for useful discussions, and to the researchers who shared their archival data so as to make this project possible, including M. Phillips, M. Hamuy, R. Kirshner, C. Li, and Y. L. Qiu. W. Li is remembered for his friendship, his tireless and excellent work on LOSS, and his many contributions to our understanding of SNe.

Some of the data presented herein were obtained at the W. M. Keck Observatory, which is operated as a scientific partnership among the California Institute of Technology, the University of California, and NASA; the observatory was made possible by the generous financial support of the W. M. Keck Foundation. KAIT and its ongoing operation were made possible by donations from Sun Microsystems, Inc., the Hewlett-Packard Company, AutoScope Corporation, Lick Observatory, the National Science Foundation (NSF), the University of California, the Sylvia and Jim Katzman Foundation, and the
TABASGO Foundation. Research at Lick Observatory is partially supported by a generous gift from Google. Some of the data presented herein were collected with the Copernico telescope (Asiago, Italy) of the INAF - Osservatorio Astronomico di Padova and the Galileo telescope (Asiago, Italy) of the Dipartimento di Fisica e Astronomia - Padova University. This research has made use of the NASA/IPAC Extragalactic Database (NED) which is operated by the Jet Propulsion Laboratory, California Institute of Technology, under contract with NASA. IRAF is distributed by the National Optical Astronomy Observatory, which is operated by the Association of Universities for Research in Astronomy (AURA) under a cooperative agreement with the NSF.

A.V.F.'s SN group at UC Berkeley has received generous financial assistance from the Christopher R. Redlich Fund, the TABASGO Foundation, and NSF grant AST1211916. M. Modjaz and the SNYU group are supported in part by NSF CAREER award AST-1352405 and by NSF award AST-1413260. Y. Liu is supported in part by a NYU/CCPP James Arthur Graduate Fellowship. J. M. Silverman is supported by an NSF Astronomy and Astrophysics Postdoctoral Fellowship under award AST-1302771. R.J.F. gratefully acknowledges support from NSF grant AST-1518052, the Alfred P. Sloan Foundation, and the David and Lucile Packard Foundation. O.G. is supported in part by NSF award AST-1413260 and by an NSF Astronomy and Astrophysics Fellowship under award AST-1602595. S. Benetti and A. Pastorello are partially supported by the PRIN-INAF 2014 project Transient Universe: unveiling new types of stellar explosions with PESSTO.

\section{REFERENCES}

Aazami, A. B., \& Li, W. D. 2001, IAUC, 7568

Allington-Smith, J., et al. 1994, PASP, 106, 983

Andersen, J., et al. 1995, The Messenger, 79, 12

Anderson, J. P., Covarrubias, R. A., James, P. A., Hamuy, M., \& Habergham, S. M. 2010, MNRAS, 407, 2660

Anderson, J. P., et al. 2014, ApJ, 786, 67

-. 2016, A\&A, 589, A110

Arcavi, I., et al. 2010, ApJ, 721, 777

-. 2012, ApJL, 756, L30

Armstrong, M., Singer, D., Burket, J., \& Li, W. 2004, IAUC, 8335,1

Arnett, W. D., Bahcall, J. N., Kirshner, R. P., \& Woosley, S. E. 1989, ARA\&A, 27, 629

Baek, M., \& Li, W. 2005, IAUC, 8641

Barbon, R., Ciatti, F., \& Rosino, L. 1979, A\&A, 72, 287

Beckmann, S., \& Li, W. D. 2001, IAUC, 7564, 2

Benetti, S., Elias-Rosa, N., Blanc, G., Navasardyan, H., Turatto, M., Zampieri, L., Cappellaro, E., \& Pedani, M. 2004, IAUC, 8312

Benetti, S., et al. 2005, ApJ, 623, 1011

Berlind, P., Matheson, T., \& Calkins, M. 2003, IAUC, 8091, 2
Bersten, M. C., et al. 2012, ApJ, 757, 31

Bianco, F. B., et al. 2014, ApJS, 213, 19

Bilinski, C., Smith, N., Li, W., Williams, G. G., Zheng, W., \& Filippenko, A. V. 2015, MNRAS, 450, 246

Blanco, V. M., et al. 1987, ApJ, 320, 589

Blondin, S., Modjaz, M., Kirshner, R., Challis, P., \& Calkins, M. 2006, Central Bureau Electronic Telegrams, 526

Blondin, S., \& Tonry, J. L. 2007, ApJ, 666, 1024

Blondin, S., et al. 2012, AJ, 143, 126

Branch, D., Dang, L. C., \& Baron, E. 2009, PASP, 121, 238

Branch, D., Jeffery, D. J., Young, T. R., \& Baron, E. 2006, PASP, 118, 791

Buzzoni, B., et al. 1984, The Messenger, 38, 9

Cano, Z. 2013, MNRAS, 434, 1098

Cao, Y., Kulkarni, S. R., Gal-Yam, A., Papadogiannakis, S., Nugent, P. E., Masci, F. J., \& Bue, B. D. 2016, ApJ, 832, 86 Cao, Y., et al. 2013, ApJL, 775, L7

-. 2015, Nature, 521, 328

Chornock, R., Filippenko, A. V., Branch, D., Foley, R. J., Jha, S., \& Li, W. 2006, PASP, 118, 722

Chornock, R., et al. 2011, ApJ, 739, 41 
Chugai, N. N. 1991, MNRAS, 250, 513

Clocchiatti, A., Wheeler, J. C., Brotherton, M. S., Cochran, A. L., Wills, D., Barker, E. S., \& Turatto, M. 1996, ApJ, 462, 462

Crowther, P. A. 2007, ARA\&A, 45, 177

Dekker, H., Delabre, B., \& Dodorico, S. 1986, in Proc. SPIE, Vol. 627, Instrumentation in astronomy VI, ed. D. L. Crawford, 339-348

Dessart, L., Hillier, D. J., Li, C., \& Woosley, S. 2012, MNRAS, 424,2139

Dessart, L., Hillier, D. J., Livne, E., Yoon, S.-C., Woosley, S., Waldman, R., \& Langer, N. 2011, MNRAS, 414, 2985

Drout, M. R., et al. 2011, ApJ, 741, 97

Dudley, C. C., \& Fischer, J. 2004, Central Bureau Electronic Telegrams, 57, 1

Eldridge, J. J., Fraser, M., Smartt, S. J., Maund, J. R., \& Crockett, R. M. 2013, MNRAS, 436, 774

Elias-Rosa, N., et al. 2006, MNRAS, 369, 1880

Elmhamdi, A., Danziger, I. J., Branch, D., Leibundgut, B., Baron, E., \& Kirshner, R. P. 2006, A\&A, 450, 305

Fabricant, D., Cheimets, P., Caldwell, N., \& Geary, J. 1998, PASP, 110,79

Faran, T., et al. 2014a, MNRAS, 445, 554

-. 2014b, MNRAS, 442, 844

Filippenko, A. V. 1982, PASP, 94, 715

-. 1988, AJ, 96, 1941

-. 1989, AJ, 97, 726

Filippenko, A. V. 1991, in European Southern Observatory Conference and Workshop Proceedings, Vol. 37, European Southern Observatory Conference and Workshop Proceedings, ed. I. J. Danziger \& K. Kjaer, 343

-. 1997, ARA\&A, 35, 309

Filippenko, A. V. 2003, in From Twilight to Highlight: The Physics of Supernovae, ed. W. Hillebrandt \& B. Leibundgut, 171

Filippenko, A. V. 2005, in Astronomical Society of the Pacific Conference Series, Vol. 332, The Fate of the Most Massive Stars, ed. R. Humphreys \& K. Stanek, 34

Filippenko, A. V., \& Chornock, R. 2001, IAUC, 7638, 1

Filippenko, A. V., Chornock, R., \& Modjaz, M. 2001a, IAUC, 7579,2

Filippenko, A. V., Chornock, R., Swift, B., Modjaz, M., Simcoe, R., \& Rauch, M. 2003, IAUC, 8159

Filippenko, A. V., Desroches, L., Ganeshalingam, M., Chornock, R., \& Serduke, F. J. D. 2004, IAUC, 8331

Filippenko, A. V., \& Foley, R. J. 2005, Central Bureau Electronic Telegrams, 274, 1

Filippenko, A. V., Li, W. D., Treffers, R. R., \& Modjaz, M. 2001b, in Astronomical Society of the Pacific Conference Series, Vol. 246, IAU Colloq. 183: Small Telescope Astronomy on Global Scales, ed. B. Paczynski, W.-P. Chen, \& C. Lemme, 121

Filippenko, A. V., Matheson, T., \& Ho, L. C. 1993, ApJL, 415, L103

Filippenko, A. V., Porter, A. C., \& Sargent, W. L. W. 1990a, AJ, 100, 1575

Filippenko, A. V., \& Shields, J. C. 1990, IAUC, 5111

Filippenko, A. V., Shields, J. C., \& Richmond, M. W. 1990b, IAUC, 5069

Filippenko, A. V., et al. 1995, ApJL, 450, L11

Folatelli, G., et al. 2006, ApJ, 641, 1039

-. 2013, ApJ, 773, 53

Foley, R. J. 2015, MNRAS, 452, 2463

Foley, R. J., \& Filippenko, A. V. 2002, IAUC, 8031, 4

Foley, R. J., \& Mandel, K. 2013, ApJ, 778, 167

Foley, R. J., Silverman, J. M., Moore, M., \& Filippenko, A. V. 2006, Central Bureau Electronic Telegrams, 604, 1
Foley, R. J., Wong, D. S., Ganeshalingam, M., Filippenko, A. V., \& Chornock, R. 2004a, IAUC, 8339, 2

Foley, R. J., Wong, D. S., Moore, M., \& Filippenko, A. V. 2004b, IAUC, 8353,3

Foley, R. J., et al. 2003, PASP, 115, 1220

-. 2009, AJ, 138, 376

—. 2013, ApJ, 767, 57

Ganeshalingam, M., et al. 2010, ApJS, 190, 418

—. 2012, ApJ, 751, 142

Garavini, G., et al. 2004, AJ, 128, 387

—. 2005, AJ, 130, 2278

Garnett, D. R. 2002, ApJ, 581, 1019

Georgy, C., Meynet, G., Walder, R., Folini, D., \& Maeder, A. 2009, A\&A, 502, 611

Graham, J., Li, W., (Loss/Kait), Trondal, O., \& Schwartz, M. 2005, IAUC, 8467, 1

Graur, O., Bianco, F. B., Huang, S., Modjaz, M., Shivvers, I., Filippenko, A. V., \& Li, W. 2016a, ArXiv e-prints

Graur, O., Bianco, F. B., \& Modjaz, M. 2015, MNRAS, 450, 905

Graur, O., Bianco, F. B., Modjaz, M., Shivvers, I., Filippenko, A. V., Li, W., \& Smith, N. 2016b, ArXiv e-prints

Graur, O., \& Maoz, D. 2013, MNRAS, 430, 1746

Groh, J. H., Georgy, C., \& Ekström, S. 2013a, A\&A, 558, L1

Groh, J. H., Meynet, G., Georgy, C., \& Ekström, S. 2013b, A\&A, 558, A131

Guillochon, J., Parrent, J., \& Margutti, R. 2016, ArXiv e-prints

Hachinger, S., Mazzali, P. A., Taubenberger, S., Hillebrandt, W., Nomoto, K., \& Sauer, D. N. 2012, MNRAS, 422, 70

Hamuy, M. 2003, IAUC, 8103, 2

Hamuy, M., Maza, J., \& Morrell, N. 2005, Central Bureau Electronic Telegrams, 321, 1

Hamuy, M., \& Roth, M. 2003, IAUC, 8228, 2

Hamuy, M., \& Suntzeff, N. B. 1990, AJ, 99, 1146

Hamuy, M., et al. 2002, AJ, 124, 417

-. 2006, PASP, 118, 2

Harutyunyan, A. H., et al. 2008, A\&A, 488, 383

Howell, D. A., et al. 2005, ApJ, 634, 1190

Hutchings, D., \& Li, W. 2002, IAUC, 8026, 1

Jha, S., Matheson, T., Challis, P., Kirshner, R., \& Calkins, M. 2001, IAUC, 7566, 2

Jha, S., et al. 2000, IAUC, 7377, 2

Kasliwal, M. M., et al. 2012, ApJ, 755, 161

Kelly, P. L., \& Kirshner, R. P. 2012, ApJ, 759, 107

Kleiser, I. K. W., et al. 2011, MNRAS, 415, 372

Leaman, J., Li, W., Chornock, R., \& Filippenko, A. V. 2011, MNRAS, 412, 1419

Lee, E., et al. 2005, IAUC, 8628, 1

Leonard, D. C., Li, W., Filippenko, A. V., Foley, R. J., \& Chornock, R. 2005, ApJ, 632, 450

Leonard, D. C., et al. 2002a, AJ, 124, 2490

-. 2002b, PASP, 114, 35

Li, W., Chornock, R., Leaman, J., Filippenko, A. V., Poznanski, D., Wang, X., Ganeshalingam, M., \& Mannucci, F. 2011a, MNRAS, 412, 1473

Li, W., Filippenko, A. V., Treffers, R. R., Riess, A. G., Hu, J., \& Qiu, Y. 2001, ApJ, 546, 734

Li, W., Qui, Y., Qiao, Q., Hu, J., \& Li, Q. 1999, Science in China A: Mathematics, 42, 1075

Li, W., et al. 2011b, MNRAS, 412, 1441

Li, W. D., Chornock, R., Filippenko, A. V., \& Phillips, M. M. 2002, IAUC, 7929, 3

Li, W. D., et al. 2000, in American Institute of Physics Conference Series, Vol. 522, American Institute of Physics Conference Series, ed. S. S. Holt \& W. W. Zhang, 103-106

Liu, Y., \& Modjaz, M. 2014, ArXiv e-prints

Liu, Y.-Q., Modjaz, M., Bianco, F. B., \& Graur, O. 2016, ApJ, 827,90 
Lyman, J. D., Bersier, D., James, P. A., Mazzali, P. A., Eldridge, J. J., Fraser, M., \& Pian, E. 2016, MNRAS, 457, 328

Madison, D. R., \& Li, W. 2006, Central Bureau Electronic Telegrams, 600,1

Maoz, D., Mannucci, F., Li, W., Filippenko, A. V., Della Valle, M., \& Panagia, N. 2011, MNRAS, 412, 1508

Matheson, T., Challis, P., Kirshner, R., \& Berlind, P. 2004a, IAUC, 8311

—. 2004b, Central Bureau Electronic Telegrams, 57, 2

-. 2004c, IAUC, 8303, 1

Matheson, T., Challis, P., Kirshner, R., \& Penev, K. 2004d, IAUC, 8353,2

Matheson, T., Filippenko, A. V., Li, W., Leonard, D. C., \& Shields, J. C. 2001, AJ, 121, 1648

Matheson, T., et al. 2000, AJ, 120, 1487

—. 2008, AJ, 135, 1598

Maund, J. R., Smartt, S. J., Kudritzki, R. P., Podsiadlowski, P., \& Gilmore, G. F. 2004, Nature, 427, 129

Maund, J. R., et al. 2006, MNRAS, 369, 390

—. 2011, ApJL, 739, L37

Mazzali, P. A., et al. 2002, ApJL, 572, L61

McCray, R. 1993, ARA\&A, 31, 175

Meynet, G., \& Maeder, A. 2003, A\&A, 404, 975

Milisavljevic, D., et al. 2013, ApJ, 767, 71

Miller, J., \& Stone, R. 1993, Lick Observatory Techical Reports, 66

Modjaz, M., Kewley, L., Bloom, J. S., Filippenko, A. V., Perley, D., \& Silverman, J. M. 2011, ApJL, 731, L4

Modjaz, M., Kirshner, R., Challis, P., Blondin, S., \& Berlind, P. 2005, Central Bureau Electronic Telegrams, 342, 1

Modjaz, M., Li, W., Filippenko, A. V., King, J. Y., Leonard, D. C., Matheson, T., Treffers, R. R., \& Riess, A. G. 2001, PASP, 113,308

Modjaz, M., Liu, Y. Q., Bianco, F. B., \& Graur, O. 2016, ApJ, 832, 108

Modjaz, M., et al. 2008, AJ, 135, 1136

-. 2009, ApJ, 702, 226

-. 2014, AJ, 147, 99

Monard, L. A. G., \& Li, W. 2004, IAUC, 8350, 2

Newton, J., \& Puckett, T. 2005, Central Bureau Electronic Telegrams, 336

Oke, J. B., et al. 1995, PASP, 107, 375

Parrent, J., et al. 2007, PASP, 119, 135

Parrent, J. T., Milisavljevic, D., Soderberg, A. M., \& Parthasarathy, M. 2016, ApJ, 820, 75

Pastorello, A., Taubenberger, S., Patat, F., Benetti, S., Harutyunyan, A., Elias-Rosa, N., \& Alises, M. 2005a, IAUC, 8467, 2

Pastorello, A., et al. 2004, MNRAS, 347, 74

-. 2005b, MNRAS, 360, 950

-. 2008, MNRAS, 389, 955

-. 2012, A\&A, 537, A141

Perets, H. B., et al. 2010, Nature, 465, 322

Phillips, M. M., et al. 2007, PASP, 119, 360

Podsiadlowski, P., Joss, P. C., \& Hsu, J. J. L. 1992, ApJ, 391, 246

Poznanski, D., Prochaska, J. X., \& Bloom, J. S. 2012, MNRAS, 426, 1465

Poznanski, D., et al. 2009, ApJ, 694, 1067

Prentice, S. J., et al. 2016, MNRAS, 458, 2973

Puckett, T., Tigner, D., \& Sehgal, A. 2002, IAUC, 8037, 1

Qui, Y., Li, W., Zhao, Z., Qiao, Q., Rao, Y., Hu, J., \& Li, Q. 1999, Science in China A: Mathematics, 42, 220

Roy, R., et al. 2013, MNRAS, 434, 2032

Rubin, A., \& Gal-Yam, A. 2016, ApJ, 828, 111
Rubin, A., et al. 2016, ApJ, 820, 33

Sahu, D. K., Anupama, G. C., Srividya, S., \& Muneer, S. 2006, MNRAS, 372, 1315

Sana, H., et al. 2012, Science, 337, 444

Sanders, N. E., et al. 2015, ApJ, 799, 208

Sato, Y., Li, W. D., \& Puckett, T. 2000, IAUC, 7374, 1

Sauer, D. N., et al. 2008, MNRAS, 391, 1605

Schlafly, E. F., \& Finkbeiner, D. P. 2011, ApJ, 737, 103

Schlegel, E. M. 1990, MNRAS, 244, 269

Schmidt, G. D., Weymann, R. J., \& Foltz, C. B. 1989, PASP, 101,713

Sehgal, A., Gagliano, R., \& Puckett, T. 2006, Central Bureau Electronic Telegrams, 493, 1

Sheinis, A. I., Bolte, M., Epps, H. W., Kibrick, R. I., Miller, J. S., Radovan, M. V., Bigelow, B. C., \& Sutin, B. M. 2002, PASP, 114, 851

Silverman, J. M., et al. 2012, MNRAS, 425, 1789

Singer, D., Pugh, H., \& Li, W. 2004, IAUC, 8297, 2

Smartt, S. J. 2009, ARA\&A, 47, 63

Smartt, S. J., Eldridge, J. J., Crockett, R. M., \& Maund, J. R. 2009, MNRAS, 395, 1409

Smith, N. 2014, ARA\&A, 52, 487

Smith, N., Li, W., Filippenko, A. V., \& Chornock, R. 2011a, MNRAS, 412, 1522

Smith, N., Li, W., Silverman, J. M., Ganeshalingam, M., \& Filippenko, A. V. 2011b, MNRAS, 415, 773

Sternberg, A., et al. 2011, Science, 333, 856

Suntzeff, N., Colianni, J., Gokas, T., Winkler, F., Smith, R. C., \& Filippenko, A. V. 2001, IAUC, 7576, 5

Swift, B., \& Li, W. 2003, IAUC, 8090, 1

Swift, B., Li, W. D., \& Filippenko, A. V. 2001, IAUC, 7618, 1

Taddia, F., et al. 2012, A\&A, 537, A140

-. 2015, A\&A, 574, A60

-. 2016a, A\&A, 592, A89

-. 2016b, A\&A, 588, A5

Tomasella, L., et al. 2014, Astronomische Nachrichten, 335, 841

Tominaga, N., et al. 2005, ApJL, 633, L97

Tonry, J., \& Davis, M. 1979, AJ, 84, 1511

Valenti, S., et al. 2011, MNRAS, 416, 3138

-. 2016, MNRAS, 459, 3939

Van Dyk, S. D., Li, W., Filippenko, A. V., Humphreys, R. M., Chornock, R., Foley, R., \& Challis, P. M. 2006, ArXiv Astrophysics e-prints

Van Dyk, S. D., Peng, C. Y., King, J. Y., Filippenko, A. V., Treffers, R. R., Li, W., \& Richmond, M. W. 2000, PASP, 112, 1532

Van Dyk, S. D., et al. 2011, ApJL, 741, L28

Wagner, R. M., et al. 2004, PASP, 116, 326

Wang, X., et al. 2009a, ApJL, 699, L139

-. 2009b, ApJ, 697, 380

Wellons, S., Soderberg, A. M., \& Chevalier, R. A. 2012, ApJ, 752,17

Wheeler, J. C., \& Harkness, R. P. 1990, Reports on Progress in Physics, 53, 1467

Wheeler, J. C., Harkness, R. P., Clocchiatti, A., Benetti, S., Brotherton, M. S., Depoy, D. L., \& Elias, J. 1994, ApJL, 436, L. 135

White, C. J., et al. 2015, ApJ, 799, 52

Woosley, S. E., \& Bloom, J. S. 2006, ARA\&A, 44, 507

Yaron, O., \& Gal-Yam, A. 2012, PASP, 124, 668

Yoon, S.-C. 2015, PASA, 32, e015

Yoon, S.-C., Woosley, S. E., \& Langer, N. 2010, ApJ, 725, 940 


\section{APPENDIX}

\section{A. JOURNAL OF DATA PRESENTED HERE}

Table A1 lists every spectrum published here for the first time: a total of 151 spectra of 71 SNe. Table A2 lists all light curves rereduced from images and published here, including data for 20 SNe. See $\S 2$ for a description of the observing and data-acquisition efforts. All data will be made public via the Berkeley SNDB (http://heracles . astro . berkeley.edu/sndb), WiseREP (http://wiserep.weizmann.ac.il), and the Open Supernova Catalog (https:// sne.space/).

Table A1. Log of Spectra Published Herein

\begin{tabular}{|c|c|c|c|c|c|c|}
\hline SN Name & UT Date & File Name ${ }^{\alpha}$ & Instrument $^{\beta}$ & $\begin{array}{c}\text { WL Range } \\
(\AA)\end{array}$ & $\begin{array}{c}\text { Resolution }^{\gamma} \\
(\AA)\end{array}$ & Source $^{\beta}$ \\
\hline SN 1999an & 1999-03-10 & sn1999an-19991003.flm & OMR & $3610-8580$ & 10 & NAOC \\
\hline SN 1999br & 1999-04-24.0 & sn1999br-19990424-opt.flm & Kast & $4300-7000$ & $6 / 5$ & $\mathrm{UCB}$ \\
\hline SN 1999bu & 1999-04-18.21 & sn1999bu-19990418.flm & FAST & $3720-7540$ & 7 & CfA \\
\hline SN 1999cd & 1999-05-15.32 & sn1999cd-19990515.flm & FAST & $3720-7540$ & 7 & CfA \\
\hline SN 1999cd & 1999-05-16.27 & sn1999cd-19990516.flm & FAST & $3720-7540$ & 7 & CfA \\
\hline SN 1999el & 1999-11-05.0 & sn1999el-19991105-ui.flm & Kast & $3380-10,460$ & $6 / 11$ & $\mathrm{UCB}$ \\
\hline SN 1999gi & $1999-12-10.0$ & sn1999gi-19991210.flm & Kast & $3720-7540$ & $6 / 5$ & $\mathrm{UCB}$ \\
\hline SN 1999gi & $1999-12-12.0$ & sn1999gi-19991212.flm & Kast & $3720-7540$ & $6 / 5$ & $\mathrm{UCB}$ \\
\hline SN 1999gi & 1999-12-13.0 & sn1999gi-19991213.flm & Kast & $3720-7540$ & $6 / 5$ & $\mathrm{UCB}$ \\
\hline SN 1999gi & 2000-01-05.0 & sn1999gi-20000105.flm & Kast & $3720-7540$ & $6 / 5$ & $\mathrm{UCB}$ \\
\hline SN 1999gi & 2000-01-10.0 & sn1999gi-20000110.flm & Kast & $3720-7540$ & $6 / 5$ & $\mathrm{UCB}$ \\
\hline SN 1999gi & $2000-01-13.0$ & sn1999gi-20000113.flm & Kast & $3720-7540$ & $6 / 5$ & $\mathrm{UCB}$ \\
\hline SN 1999gi & 2000-03-04.0 & sn1999gi-20000304.flm & Kast & $3720-7540$ & $6 / 5$ & $\mathrm{UCB}$ \\
\hline SN 1999gi & 2000-03-08.0 & sn1999gi-20000308.flm & Kast & $3720-7540$ & $6 / 5$ & $\mathrm{UCB}$ \\
\hline SN 1999gi & $2000-03-15.0$ & sn1999gi-20000315-ui.flm & Kast & $3300-10,500$ & $6 / 11$ & $\mathrm{UCB}$ \\
\hline SN 1999gi & $2000-03-25.0$ & sn1999gi-20000325-opts.flm & LRIS & $4380-6840$ & 7 & $\mathrm{UCB}$ \\
\hline SN 1999gi & $2000-03-29.0$ & sn1999gi-20000329-ui.flm & Kast & $3300-10,500$ & $6 / 11$ & $\mathrm{UCB}$ \\
\hline SN 1999gi & $2000-04-25.0$ & sn1999gi-20000425.flm & Kast & $3720-7540$ & $6 / 5$ & $\mathrm{UCB}$ \\
\hline SN 1999gi & $2000-04-27.0$ & sn1999gi-20000427-ui.flm & Kast & $3300-10,350$ & $6 / 11$ & $\mathrm{UCB}$ \\
\hline SN 1999gi & $2000-05-10.0$ & sn1999gi-20000510.flm & Kast & $3720-7540$ & $6 / 5$ & $\mathrm{UCB}$ \\
\hline SN 1999gi & 2000-05-26.0 & sn1999gi-20000526.flm & Kast & $3720-7540$ & $6 / 5$ & $\mathrm{UCB}$ \\
\hline SN 1999go & $1999-12-28$ & 1999go_19991228.flm & DFOSC & $3330-9040$ & 14 & Asiago \\
\hline SN 1999go & 1999-12-29 & 1999go_19991229.flm & DFOSC & 3330-9040 & 14 & Asiago \\
\hline SN 1999go & 2000-01-02.23 & sn1999go-20000102.flm & FAST & $3720-7540$ & 7 & CfA \\
\hline SN $2000 \mathrm{C}$ & $2000-01-25$ & 2000C_20000125.flm & $\mathrm{B} \& \mathrm{C}_{1.2}$ & $4490-9090$ & 22 & Asiago \\
\hline SN $2000 \mathrm{C}$ & $2000-01-27$ & 2000C_20000127.flm & AFOSC & $3520-7560$ & 18 & Asiago \\
\hline SN 2000C & 2000-01-28.14 & sn2000c-20000128.flm & FAST & $3720-7540$ & 7 & CfA \\
\hline SN $2000 \mathrm{C}$ & 2000-01-29 & 2000C_20000129.flm & AFOSC & $3520-7490$ & 18 & Asiago \\
\hline SN $2000 \mathrm{C}$ & 2000-01-29.20 & sn2000c-20000129.flm & FAST & $3720-7540$ & 7 & CfA \\
\hline SN $2000 \mathrm{C}$ & 2000-02-01 & 2000C_20000201.flm & AFOSC & $3550-7490$ & 18 & Asiago \\
\hline SN 2000C & $2000-02-05.25$ & sn2000c-20000205.flm & FAST & $3720-7540$ & 7 & CfA \\
\hline SN $2000 \mathrm{C}$ & 2000-02-11 & 2000C_20000211.flm & AFOSC & $3620-7590$ & 18 & Asiago \\
\hline SN 2000L & 2000-03-01.29 & sn20001-20000301.flm & FAST & $3720-7540$ & 7 & CfA \\
\hline SN 2000L & $2000-03-15.0$ & sn20001-20000315-ui.flm & Kast & $3300-10,500$ & $6 / 11$ & $\mathrm{UCB}$ \\
\hline SN $2000 \mathrm{~N}$ & 2000-03-08.43 & sn2000n-20000308.flm & FAST & $3720-7540$ & 7 & CfA \\
\hline
\end{tabular}


TABLE A1 - continued from previous page

\begin{tabular}{|c|c|c|c|c|c|c|}
\hline SN Name & UT Date & File Name ${ }^{\alpha}$ & Instrument $^{\beta}$ & $\begin{array}{c}\text { WL Range } \\
(\AA)\end{array}$ & $\begin{array}{c}\text { Resolution }^{\gamma} \\
(\AA)\end{array}$ & Source $^{\beta}$ \\
\hline SN $2000 N$ & 2000-03-09 & 2000N_20000309.flm & DFOSC & $3850-6840$ & 6 & Asiago \\
\hline SN $2000 N$ & 2000-03-13 & 2000N_20000313.flm & DFOSC & $3550-9040$ & 11 & Asiago \\
\hline SN 2000el & $2000-11-29.0$ & sn2000el-20001129-ui.flm & Kast & $3300-10,450$ & $6 / 11$ & $\mathrm{UCB}$ \\
\hline SN 2000eo & $2000-12-21.0$ & sn2000eo-20001221-ur.flm & Kast & $3250-7810$ & $6 / 5$ & UCB \\
\hline SN 2000ex & 2000-11-28.29 & sn2000ex-20001128.flm & FAST & $3760-7540$ & 7 & CfA \\
\hline SN 2000ex & 2000-11-29.0 & sn2000ex-20001129-ur.flm & Kast & $3300-7800$ & $6 / 5$ & $\mathrm{UCB}$ \\
\hline SN 2001J & 2001-01-19.45 & sn2001j-20010119.flm & FAST & $3720-7540$ & 7 & CfA \\
\hline SN $2001 K$ & 2001-03-30.0 & sn2001k-20010330-ur.flm & Kast & $3300-7800$ & $6 / 5$ & $\mathrm{UCB}$ \\
\hline SN 2001M & 2001-02-01.0 & sn2001m-20010201-ui.flm & Kast & $3260-10,600$ & $6 / 11$ & $\mathrm{UCB}$ \\
\hline SN 2001Q & 2001-02-01.0 & sn2001q-20010201-ui.flm & Kast & $3260-10,600$ & $6 / 11$ & UCB \\
\hline SN 2001ac & 2001-03-14.45 & sn2001ac-20010314.flm & FAST & $3720-7540$ & 7 & CfA \\
\hline SN 2001ac & 2001-03-25.36 & sn2001ac-20010325-blue-mmt.flm & MMT-Blue & $3250-8850$ & 8 & CfA \\
\hline SN 2001ci & 2001-05-30.285 & sn2001ci-com-20010530.285-joined.flm & ESI & $3920-10,190$ & 0.5 & UCB \\
\hline SN 2001fz & 2001-11-19.50 & sn2001fz-20011119.flm & FAST & $3720-7540$ & 7 & CfA \\
\hline SN 2001hf & 2002-01-14.0 & sn2001hf-20020114-ur.flm & Kast & $3260-7940$ & $6 / 5$ & $\mathrm{UCB}$ \\
\hline SN 2001is & 2002-01-06.29 & sn2001is-20020106.flm & FAST & $3720-7540$ & 7 & CfA \\
\hline SN 2001is & 2002-01-07.31 & sn2001is-20020107.flm & FAST & $3720-7540$ & 7 & CfA \\
\hline SN 2001is & 2002-01-08 & 2001is_20020108.flm & AFOSC & $3360-7720$ & 25 & Asiago \\
\hline SN 2001is & 2002-01-09 & 2001is_20020109.flm & AFOSC & $3360-7720$ & 25 & Asiago \\
\hline SN 2001is & 2002-01-14.0 & sn2001is-20020114-ui.flm & Kast & $3260-10,570$ & $6 / 11$ & $\mathrm{UCB}$ \\
\hline SN 2002J & 2002-02-11.0 & sn2002j-20020211-ui.flm & Kast & $3300-10,400$ & $6 / 11$ & $\mathrm{UCB}$ \\
\hline SN 2002J & $2002-02-14.0$ & sn2002j-20020214-os.flm & LRIS & $3950-8830$ & 7 & UCB \\
\hline SN 2002ce & 2002-04-12.18 & sn2002ce-20020412.flm & FAST & $3720-7520$ & 7 & CfA \\
\hline SN 2002ce & $2002-05-07.0$ & sn2002ce-20020507-ui.flm & Kast & $3300-10,300$ & $6 / 11$ & $\mathrm{UCB}$ \\
\hline SN 2002dq & 2002-08-09.0 & sn2002dq-20020809-ui.flm & Kast & $3150-10,400$ & $6 / 11$ & $\mathrm{UCB}$ \\
\hline SN 2002gw & $2002-10-29$ & sn02gw.blue.29oct02.flm & LDSS-2 & $3600-9000$ & 13.5 & Asiago \\
\hline SN 2002jj & 2002-12-05.35 & sn2002jj-20021205.flm & FAST & $3720-7520$ & 7 & CfA \\
\hline SN 2002jj & $2002-12-12.0$ & sn2002jj-20021212-ui.flm & Kast & $3170-10,400$ & $6 / 11$ & $\mathrm{UCB}$ \\
\hline SN 2002jj & 2003-01-28.0 & sn2002jj-20030128-ui.flm & Kast & $3290-10,400$ & $6 / 11$ & $\mathrm{UCB}$ \\
\hline SN 2002jz & 2003-01-07.0 & sn2002jz-20030107-br.flm & LRIS & $3080-9430$ & 7 & $\mathrm{UCB}$ \\
\hline SN 2002jz & 2003-01-28.0 & sn2002jz-20030128-ui.flm & Kast & $3310-10,400$ & $6 / 11$ & $\mathrm{UCB}$ \\
\hline SN 2002jz & 2003-02-28.0 & sn2002jz-20030228-br.flm & LRIS & $3120-9420$ & 7 & UCB \\
\hline SN 2003E & 2003-01-28.0 & sn2003E-20030128-ui.flm & Kast & $3270-10,400$ & $6 / 11$ & $\mathrm{UCB}$ \\
\hline SN 2003G & 2003-01-28.0 & sn2003G-20030128-ui.flm & Kast & $3100-10,400$ & $6 / 11$ & $\mathrm{UCB}$ \\
\hline SN 2003G & 2003-02-04.0 & sn2003G-20030204-ui.flm & Kast & $3100-10,400$ & $6 / 11$ & $\mathrm{UCB}$ \\
\hline SN 2003G & $2003-02-28.0$ & sn2003g-20030228-br.flm & LRIS & $3040-9420$ & 7 & $\mathrm{UCB}$ \\
\hline SN $2003 \mathrm{H}$ & $2003-02-04.0$ & sn2003H-20030204-ui.flm & Kast & $3210-10,400$ & $6 / 11$ & $\mathrm{UCB}$ \\
\hline SN $2003 \mathrm{H}$ & $2003-02-28.0$ & sn2003h-20030228-br.flm & LRIS & $3170-9420$ & 7 & $\mathrm{UCB}$ \\
\hline SN 2003aa & 2003-02-04.0 & sn2003aa-20030204-ui.flm & Kast & $3120-10,400$ & $6 / 11$ & $\mathrm{UCB}$ \\
\hline SN 2003aa & $2003-02-28.0$ & sn2003aa-20030228-br.flm & LRIS & $3050-9420$ & 7 & $\mathrm{UCB}$ \\
\hline SN 2003aa & 2003-04-08.0 & sn2003aa-20030408-ui.flm & Kast & $3200-10,400$ & $6 / 11$ & $\mathrm{UCB}$ \\
\hline SN 2003aa & $2003-05-25.0$ & sn2003aa-20030525-brs.flm & LRIS & $3220-9420$ & 7 & UCB \\
\hline SN 2003ao & 2003-02-22.31 & sn2003ao-20030222.flm & FAST & $3720-7540$ & 7 & CfA \\
\hline SN 2003bk & 2003-03-04 & 2003bk_20030304_E3p6_EFOSC2.flm & EFOSC & $3380-10,030$ & 14 & Asiago \\
\hline
\end{tabular}


TABLE A1 - continued from previous page

\begin{tabular}{|c|c|c|c|c|c|c|}
\hline SN Name & UT Date & File Name ${ }^{\alpha}$ & Instrument $^{\beta}$ & $\begin{array}{c}\text { WL Range } \\
(\AA)\end{array}$ & $\begin{array}{c}\text { Resolution }^{\gamma} \\
(\AA) \\
\end{array}$ & Source $^{\beta}$ \\
\hline SN 2003br & 2003-03-11.48 & sn2003br-20030311.flm & FAST & $3720-7540$ & 7 & CfA \\
\hline SN 2003dr & 2003-06-29.0 & sn2003dr-20030629-brs.flm & LRIS & $3250-9200$ & 7 & $\mathrm{UCB}$ \\
\hline SN 2003dv & 2003-07-06.366 & sn2003dv-20030706.366-ui.flm & Kast & $3300-10,500$ & $6 / 11$ & $\mathrm{UCB}$ \\
\hline SN 2003ed & $2003-05-25.0$ & sn2003ed-20030525-brs.flm & LRIS & $3220-9420$ & 7 & $\mathrm{UCB}$ \\
\hline SN 2003ed & 2003-05-30.0 & sn2003ed-20030530-ui.flm & Kast & $3230-10,400$ & $6 / 11$ & $\mathrm{UCB}$ \\
\hline SN 2003ed & 2003-06-07.378 & sn2003ed-20030607.378-ui.flm & Kast & $3600-10,300$ & $6 / 11$ & UCB \\
\hline SN 2003ed & 2003-07-06.329 & sn2003ed-20030706.329-ui.flm & Kast & $3350-10,310$ & $6 / 11$ & UCB \\
\hline SN 2003ed & 2003-09-03.0 & sn2003ed-20030903-ui.flm & Kast & $3250-10,400$ & $6 / 11$ & $\mathrm{UCB}$ \\
\hline SN 2003ef & 2003-05-21.19 & sn2003ef-20030521.flm & FAST & $3720-7540$ & 7 & CfA \\
\hline SN 2003id & 2003-09-19.41 & sn2003id-20030919.flm & FAST & $3720-7540$ & 7 & CfA \\
\hline SN 2003id & 2003-10-23.432 & sn2003id-20031023.432-ui.flm & Kast & $3830-10,500$ & $6 / 11$ & $\mathrm{UCB}$ \\
\hline SN 2003ld & 2004-01-13.16 & sn2003ld-20040113.flm & FAST & $3720-7540$ & 7 & CfA \\
\hline SN $2004 \mathrm{C}$ & 2004-01-15.50 & sn2004c-20040115.flm & FAST & $3720-7540$ & 7 & CfA \\
\hline SN 2004C & 2004-01-17.0 & sn2004c-20040117-ui.flm & Kast & $3320-10,400$ & $6 / 11$ & $\mathrm{UCB}$ \\
\hline SN $2004 \mathrm{C}$ & 2004-01-18.49 & sn2004c-20040118-mmt.flm & MMT-Blue & $3270-8460$ & 8 & CfA \\
\hline SN $2004 \mathrm{C}$ & 2004-01-18.49 & sn2004c-20040118.flm & MMT-Blue & $3720-7540$ & 8 & CfA \\
\hline SN $2004 \mathrm{C}$ & 2004-01-19.44 & sn2004c-20040119.flm & FAST & $3720-7520$ & 7 & CfA \\
\hline SN $2004 \mathrm{C}$ & 2004-03-16.599 & sn2004c-20040316.599-br.flm & LRIS & $3350-9230$ & 7 & UCB \\
\hline SN $2004 \mathrm{C}$ & 2004-03-17.40 & sn2004c-20040317.flm & FAST & $3720-7540$ & 7 & CfA \\
\hline SN 2004C & 2004-04-26.336 & sn2004c-20040426.336-br.flm & LRIS & $3150-9410$ & 7 & UCB \\
\hline SN $2004 \mathrm{C}$ & 2004-11-14.0 & sn2004c-20041114-br.flm & LRIS & $3070-9400$ & 7 & $\mathrm{UCB}$ \\
\hline SN 2004al & 2004-03-13.26 & sn2004al-20040313.flm & FAST & $3720-7540$ & 7 & CfA \\
\hline SN 2004aq & 2004-03-16.618 & sn2004aq-20040316.618-br.flm & LRIS & $3420-9230$ & 7 & $\mathrm{UCB}$ \\
\hline SN 2004be & 2004-04-13.22 & sn2004be-20040413.flm & FAST & $3720-7540$ & 7 & CfA \\
\hline SN 2004bm & 2004-05-12.226 & sn2004bm-20040512.226-ui.flm & Kast & $3390-10,580$ & $6 / 11$ & $\mathrm{UCB}$ \\
\hline SN 2004cc & 2004-06-13.241 & sn2004cc-20040613.241-ui.flm & Kast & $3320-10,400$ & $6 / 11$ & $\mathrm{UCB}$ \\
\hline SN 2004cc & $2004-06-20.0$ & sn2004cc-20040620-ui.flm & Kast & $3300-10,400$ & $6 / 11$ & $\mathrm{UCB}$ \\
\hline SN 2004ci & 2004-06-20.0 & sn2004ci-20040620-ui.flm & Kast & $3300-10,400$ & $6 / 11$ & $\mathrm{UCB}$ \\
\hline SN 2004dd & $2004-07-18.0$ & sn2004dd-20040718-ui.flm & Kast & $3300-10,400$ & $6 / 11$ & $\mathrm{UCB}$ \\
\hline SN 2004dk & 2004-08-08.0 & sn2004dk-20040808-ui.flm & Kast & $3310-10,400$ & $6 / 11$ & $\mathrm{UCB}$ \\
\hline SN 2004dk & 2004-08-16.174 & sn2004dk-20040816.174-ui.flm & Kast & $3320-10,400$ & $6 / 11$ & $\mathrm{UCB}$ \\
\hline SN $2004 \mathrm{dk}$ & 2004-09-10.154 & sn2004dk-20040910.154-ui.flm & Kast & $3310-10,500$ & $6 / 11$ & $\mathrm{UCB}$ \\
\hline SN 2004dk & 2004-09-24.15 & sn2004dk-20040924.150-ui.flm & Kast & $3320-10,500$ & $6 / 11$ & $\mathrm{UCB}$ \\
\hline SN 2004dk & $2005-05-11.568$ & sn2004dk-20050511.568-br.flm & LRIS & $3100-9350$ & 7 & UCB \\
\hline SN 2004er & 2004-10-24 & SN04er_b01_CLA_LD_24oct04.flm & LDSS-2 & $3600-8990$ & 13.5 & CSP \\
\hline SN 2004fc & $2004-12-17.168$ & sn2004fc-20041217.168-ui.flm & Kast & $3320-10,600$ & $6 / 11$ & $\mathrm{UCB}$ \\
\hline SN 2004fx & 2004-11-14.0 & sn2004fx-20041114-br.flm & LRIS & $3080-9400$ & 7 & $\mathrm{UCB}$ \\
\hline SN 2004fx & 2004-12-17.342 & sn2004fx-20041217.342-ui.flm & Kast & $3340-10,550$ & $6 / 11$ & $\mathrm{UCB}$ \\
\hline SN 2004gq & $2004-12-12.482$ & sn2004gq-20041212.482-br.flm & LRIS & $3200-9320$ & 7 & $\mathrm{UCB}$ \\
\hline SN 2004gq & $2004-12-13$ & SN04gq_b01_DUP_WF_13dec04.flm & WFCCD & $3800-9230$ & 6 & CSP \\
\hline SN 2004gq & 2004-12-17.317 & sn2004gq-20041217.317-ui.flm & Kast & $3370-10,300$ & $6 / 11$ & $\mathrm{UCB}$ \\
\hline SN 2004gq & 2005-01-16.287 & sn2004gq-20050116.287-ui.flm & Kast & $3300-10,500$ & $6 / 11$ & $\mathrm{UCB}$ \\
\hline SN 2004gq & 2005-02-12.395 & sn2004gq-20050212.395-br.flm & LRIS & $3780-9250$ & 7 & $\mathrm{UCB}$ \\
\hline SN 2004gq & 2005-03-11.29 & sn2004gq-20050311.290-br.flm & LRIS & $3400-9260$ & 7 & UCB \\
\hline
\end{tabular}


TABLE A1 - continued from previous page

\begin{tabular}{|c|c|c|c|c|c|c|}
\hline SN Name & UT Date & File Name ${ }^{\alpha}$ & Instrument $^{\beta}$ & $\begin{array}{c}\text { WL Range } \\
(\AA)\end{array}$ & $\begin{array}{c}\text { Resolution }^{\gamma} \\
(\AA)\end{array}$ & Source $^{\beta}$ \\
\hline SN 2005E & 2005-01-15.353 & sn2005e-20050115.353-br.flm & LRIS & $3380-9250$ & 7 & $\mathrm{UCB}$ \\
\hline SN 2005E & 2005-02-01.125 & sn2005e-20050201.125-ui.flm & Kast & $3310-10,500$ & $6 / 11$ & $\mathrm{UCB}$ \\
\hline SN $2005 \mathrm{E}$ & $2005-03-11.257$ & sn2005e-20050311.257-br.flm & LRIS & $3400-9260$ & 7 & $\mathrm{UCB}$ \\
\hline SN $2005 \mathrm{H}$ & 2005-01-17.113 & sn2005h-20050117.113-ui.flm & Kast & $3300-10,500$ & $6 / 11$ & $\mathrm{UCB}$ \\
\hline SN $2005 \mathrm{H}$ & 2005-03-11.238 & sn2005h-20050311.238-br.flm & LRIS & $3390-9260$ & 7 & $\mathrm{UCB}$ \\
\hline SN 2005J & 2005-02-04 & SN05J_b01_DUP_WF_04feb05.flm & WFCCD & $3800-9230$ & 6 & CSP \\
\hline SN 2005ad & 2005-03-11.222 & sn2005ad-20050311.222-br.flm & LRIS & $3400-9260$ & 7 & $\mathrm{UCB}$ \\
\hline SN 2005an & $2005-03-15$ & SN05an_b01_DUP_WF_15mar05.flm & WFCCD & 3800-9230 & 6 & CSP \\
\hline SN 2005aq & $2005-03-11.284$ & sn2005aq-20050311.284-br.flm & LRIS & $3400-9260$ & 7 & $\mathrm{UCB}$ \\
\hline SN 2005bb & 2005-04-07 & SN05bb_b01_DUP_WF_07apr05.flm & WFCCD & $3800-9230$ & 6 & CSP \\
\hline SN 2005io & 2005-11-05.636 & sn2005io-20051105.636-br.flm & LRIS & $3200-9240$ & 7 & $\mathrm{UCB}$ \\
\hline SN 2005lr & $2005-12-18$ & SN05lr_g01_NTT_EM_18dec05.flm & EMMI & $4000-10,200$ & 9 & CSP \\
\hline SN 2005lr & $2005-12-20$ & SN05lr_b01_DUP_WF_20dec05.flm & WFCCD & $3800-9230$ & 6 & CSP \\
\hline SN 2005mg & $2005-12-28$ & sn2005mg-20051228.flm.gif.traced.flm ${ }^{\delta}$ & FAST & $3490-7400$ & 7 & CfA \\
\hline SN $2005 \mathrm{mg}$ & $2005-12-29.12$ & sn2005mg-20051229.flm.gif.traced.flm ${ }^{\delta}$ & FAST & $3490-7420$ & 7 & CfA \\
\hline SN $2005 \mathrm{mg}$ & 2006-01-05.145 & sn2005mg-20060105.145-ui.flm & Kast & $3310-10,500$ & $6 / 11$ & UCB \\
\hline SN $2006 \mathrm{~F}$ & $2006-01-16$ & SN06F_g01_NTT_EM_16jan06.flm & EMMI & $4000-10,200$ & 9 & CSP \\
\hline SN $2006 \mathrm{~T}$ & 2006-02-13 & SN06T_g01_NTT_EM_13feb06.flm & EMMI & $4000-10,200$ & 9 & CSP \\
\hline SN 2006T & $2006-02-22.32$ & sn2006t-20060222.320-ui.flm & Kast & $3320-10,300$ & $6 / 11$ & UCB \\
\hline SN 2006be & $2006-03-30$ & SN06be_b01_DUP_WF_30mar06.flm & WFCCD & $3800-9230$ & 6 & CSP \\
\hline SN 2006be & 2006-05-05.419 & sn2006be-20060505.419-ui.flm & Kast & $3350-10,400$ & $6 / 11$ & $\mathrm{UCB}$ \\
\hline SN 2006bp & $2006-04-28.26$ & sn2006bp-20060428.260-ui.flm & Kast & $3320-10,500$ & $6 / 11$ & $\mathrm{UCB}$ \\
\hline SN 2006ca & 2006-05-05.399 & sn2006ca-20060505.399-ui.flm & Kast & $3350-10,400$ & $6 / 11$ & $\mathrm{UCB}$ \\
\hline SN 2006ca & 2006-06-20.367 & sn2006ca-20060620.367-ui.flm & Kast & $3330-10,580$ & $6 / 11$ & $\mathrm{UCB}$ \\
\hline SN 2006ca & 2006-07-04.363 & sn2006ca-20060704.363-ui.flm & Kast & $3310-10,700$ & $6 / 11$ & UCB \\
\hline SN 2006eg & 2006-08-24.399 & sn2006eg-20060824.399-ui.flm & Kast & $3320-10,400$ & $6 / 11$ & $\mathrm{UCB}$ \\
\hline SN 2006qr & $2006-12-01.49$ & sn2006qr-20061201.490-ui.flm & Kast & $3310-10,500$ & $6 / 11$ & $\mathrm{UCB}$ \\
\hline SN 2006qr & 2006-12-13 & SN06qr_b01_DUP_BC_13dec06.flm & $\mathrm{B} \& \mathrm{C}_{2.5}$ & 3620-9820 & 8 & $\mathrm{CSP}$ \\
\hline
\end{tabular}

${ }^{\alpha}$ Different groups utilize different naming conventions for their data; we preserve these differences and the original names of these files.

${ }^{\beta}$ See $\S 2$ for a description of the instruments and observational efforts listed here.

${ }^{\gamma}$ Listed resolutions are estimates of the average resolution for the instrument (if two resolutions are given, they refer to the blue side and red side of the spectrograph separately).

${ }^{\delta}$ Traced from image of plot; see $\S 4.2 .9$. 
Table A2. Log of Light Curves Published Herein

\begin{tabular}{l|cc|cc}
\hline SN Name & Telescope & Filters & N Detections & Date Range \\
\hline SN 2000N & KAIT & clear & 9 & $2000-03-04-2000-06-03$ \\
SN 2001J & KAIT & clear & 2 & $2001-01-15-2001-01-16$ \\
SN 2001M & KAIT & clear & 4 & $2001-01-21-2001-02-03$ \\
SN 2001ci & KAIT & clear & 8 & $2001-04-25-2001-05-12$ \\
SN 2002ds & KAIT & clear & 6 & $2002-06-25-2002-07-25$ \\
SN 2002jj & KAIT & clear & 6 & $2002-10-24-2003-01-17$ \\
SN 2002jz & KAIT & clear & 6 & $2002-12-24-2003-01-31$ \\
SN 2003bk & KAIT & clear & 6 & $2003-02-28-2003-05-31$ \\
SN 2003br & KAIT & clear & 8 & $2003-03-07-2003-06-03$ \\
SN 2003bw & KAIT & clear & 4 & $2003-03-03-2003-03-28$ \\
SN 2003id & KAIT,Nickel & B,V,R,I,clear & 8 & $2003-09-16-2003-10-28$ \\
SN 2004C & KAIT & clear & 9 & $2004-01-21-2004-05-22$ \\
SN 2004al & KAIT & clear & 5 & $2004-03-03-2004-04-22$ \\
SN 2004bm & KAIT & clear & 4 & $2004-04-13-2004-05-08$ \\
SN 2004er & KAIT & clear & 15 & $2004-09-25-2005-02-07$ \\
SN 2005ci & KAIT & clear & 20 & $2005-06-10-2006-05-02$ \\
SN 2005io & KAIT & clear & 13 & $2005-11-03-2006-02-24$ \\
SN 2005lr & KAIT & clear & 5 & $2005-12-04-2006-01-06$ \\
SN 2005mg & KAIT & clear & 2 & $2005-12-29-2006-01-10$ \\
SN 2006eg & KAIT & clear & 7 & $2006-07-31-2006-10-15$ \\
\hline
\end{tabular}

